Pacific

Journal of

Mathematics

EXTENDING HECKE ENDOMORPHISM ALGEBRAS

Jie Du, BRIAn J. PARshall and LeONARD L. SCOTT

Volume 279 No. 1-2

December 2015 


\title{
EXTENDING HECKE ENDOMORPHISM ALGEBRAS
}

\author{
Jie Du, Brian J. PARshall and Leonard L. ScotT
}

We dedicate this paper to the memory of Robert Steinberg.

\begin{abstract}
The (Iwahori-)Hecke algebra in the title is a $q$-deformation $\mathcal{H}$ of the group algebra of a finite Weyl group $W$. The algebra $\mathcal{H}$ has a natural enlargement to an endomorphism algebra $\mathcal{A}=\operatorname{End}_{\mathcal{H}}(\mathcal{T})$ where $\mathcal{T}$ is a $q$-permutation module. In type $A_{n}$ (i.e., $W \cong \mathfrak{S}_{n+1}$ ), the algebra $\mathcal{A}$ is a $q$-Schur algebra which is quasi-hereditary and plays an important role in the modular representation of the finite groups of Lie type. In other types, $\mathcal{A}$ is not always quasi-hereditary, but the authors conjectured 20 year ago that $\mathcal{T}$ can be enlarged to an $\mathcal{H}$-module $\mathcal{T}^{+}$so that $\mathcal{A}^{+}=\operatorname{End}_{\mathcal{H}}\left(\mathcal{T}^{+}\right)$is at least standardly stratified, a weaker condition than being quasi-hereditary, but with "strata" corresponding to Kazhdan-Lusztig two-sided cells.

The main result of this paper is a "local" version of this conjecture in the equal parameter case, viewing $\mathcal{H}$ as defined over $\mathbb{Z}\left[t, t^{-1}\right]$, with the localization at a prime ideal generated by a cyclotomic polynomial $\Phi_{2 e}(t)$, $e \neq 2$. The proof uses the theory of rational Cherednik algebras (also known as RDAHAs) over similar localizations of $\mathbb{C}\left[t, t^{-1}\right]$. In future papers, the authors hope to prove global versions of the conjecture, maintaining these localizations.
\end{abstract}

\section{Introduction}

Let $\mathscr{G}=\{G(q)\}$ be a family of finite groups of Lie type having irreducible (finite) Coxeter system $(W, S)$ [Curtis and Reiner 1987, (68.22)]. The pair $(W, S)$ remains fixed throughout this paper. Let $B(q)$ be a Borel subgroup of $G(q)$. There are index parameters $c_{s} \in \mathbb{Z}, s \in S$, defined by

$$
\left[B(q):{ }^{s} B(q) \cap B(q)\right]=q^{c_{s}}, \quad s \in S .
$$

Research supported in part by the Australian Research Council and National Science Foundation. MSC2010: primary 20C08, 20C33; secondary 16S50, 16S80.

Keywords: Hecke algebra, Cherednik, root of unity, stratified, quasi-hereditary. 
The generic Hecke algebra $\mathcal{H}$ over the ring $\mathcal{Z}=\mathbb{Z}\left[t, t^{-1}\right]$ of Laurent polynomials associated to $\mathscr{G}$ has basis $T_{w}, w \in W$, subject to relations

$$
T_{s} T_{w}= \begin{cases}T_{s w}, & s w>w \\ t^{2 c_{s}} T_{s w}+\left(t^{2 c_{s}}-1\right) T_{w}, & s w<w\end{cases}
$$

for $s \in S, w \in W$. This algebra is defined just using $t^{2}$, but it is convenient to have its square root $t$ available. We call $\mathcal{H}$ a Hecke algebra of Lie type over $\mathcal{Z}$. It is related to the representation theory of the groups in $\mathscr{G}$ as follows: for any prime power $q$, let $R$ be any field (we will shortly allow $R$ to be a ring) in which the integer $q$ is invertible and has a square root $\sqrt{q}$. Let $\mathcal{H}_{R}=\mathcal{H} \otimes_{\mathcal{Z}} R$ be the algebra obtained by base change through the map $\mathcal{Z} \rightarrow R, t \mapsto \sqrt{q}$. Then $\mathcal{H}_{R} \cong \operatorname{End}_{G(q)}\left(\operatorname{ind}_{B(q)}^{G(q)} R\right)$. Thus, the generic Hecke algebra $\mathcal{H}$ is the quantumization (in the sense of [Deng et al. 2008, §0.4]) of an infinite family of important endomorphism algebras.

In type $A_{n}$, i.e., when $\mathscr{G}=\left\{\mathrm{GL}_{n+1}(q)\right\}$, one can also consider the $q$-Schur algebras, viz., algebras Morita equivalent to

$$
S_{R}:=\operatorname{End}_{\mathcal{H}_{R}}\left(\bigoplus_{J \subseteq S} \operatorname{ind}_{\mathcal{H}_{J, R}}^{\mathcal{H}_{R}} \mathrm{IND}_{J}\right) .
$$

In this case, $S_{R}$ is a quasi-hereditary algebra whose representation theory is closely related to that of the quantum general linear groups. The $q$-Schur algebras have historically played an important role in representation theory of the finite general linear groups, thanks to the work of Dipper, James, and others. More generally, although the definition (1.2) makes sense in all types, less is known about its properties or the precise role it plays in the representation theory or homological algebra of the corresponding groups in $\mathscr{G}$. The purpose of this paper, and its sequels, is to enhance $S_{R}$ in a way described below, so that it does become relevant to these questions.

1A. Stratifying systems. At this point, it will be useful to review the notion of a strict stratifying system for an algebra. These systems provide a framework for studying algebras similar to quasi-hereditary algebras. They appear in the statement of the first main theorem. Although the algebras in Theorem 5.6 below are shown later to be quasi-hereditary, the theory of stratifying systems is useful both in providing a framework and as a tool in obtaining the final results.

First, recall that a preorder on a set $X$ is a transitive and reflexive relation $\leq$. The associated equivalence relation $\sim$ on $X$ is defined by setting, for $x, y \in X$,

$$
x \sim y \Longleftrightarrow x \leq y \text { and } y \leq x .
$$

A preorder induces an evident partial order, still denoted $\leq$, on the set $\bar{X}$ of equivalence classes of $\sim$. In this paper, a set $X$ with a preorder is called a quasi-poset. Also, if $x \in X$, let $\bar{x} \in \bar{X}$ be its associated equivalence class. 
Now let $R$ be a Noetherian commutative ring, and let $A$ be an $R$-algebra, finitely generated and projective as an $R$-module. Let $\Lambda$ be a finite quasi-poset. For each $\lambda \in \Lambda$, it is required that there is given a finitely generated $A$-module $\Delta(\lambda)$ and a finitely generated projective $A$-module $P(\lambda)$ together with a fixed surjective morphism $P(\lambda) \rightarrow \Delta(\lambda)$ of $A$-modules. The following conditions are required:

(1) For $\lambda, \mu \in \Lambda$,

$$
\operatorname{Hom}_{A}(P(\lambda), \Delta(\mu)) \neq 0 \Rightarrow \lambda \leq \mu .
$$

(2) Every irreducible $A$-module $L$ is a homomorphic image of some $\Delta(\lambda)$.

(3) For $\lambda \in \Lambda$, the $A$-module $P(\lambda)$ has a finite filtration by $A$-submodules with top section $\Delta(\lambda)$ and other sections of the form $\Delta(\mu)$ with $\bar{\mu}>\bar{\lambda}$.

When these conditions all hold, the data $\{\Delta(\lambda)\}_{\lambda \in \Lambda}$ is a strict stratifying system for $A$-mod. It is also clear that $\Delta(\lambda)_{R^{\prime}}, P(\lambda)_{R^{\prime}}, \ldots$ is a strict stratifying system for

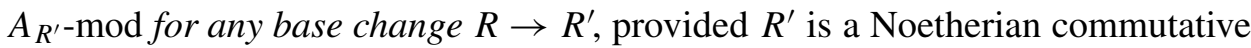
ring. (Notice that condition (2) is redundant if it is known that the direct sum of the projective modules in (3) is a progenerator - a property preserved by base change.)

An ideal $J$ in an $R$-algebra $A$ as above is called a stratifying ideal provided that $J$ is an $R$-direct summand of $A$ (or equivalently, the inclusion $J \hookrightarrow A$ is $R$-split), and for $A / J$-modules $M, N$ inflation defines an isomorphism

$$
\operatorname{Ext}_{A / J}^{n}(M, N) \stackrel{\sim}{\longrightarrow} \operatorname{Ext}_{A}^{n}(M, N), \quad \text { for all } n \geq 0 .
$$

of Ext-groups. A standard stratification of length $n$ of $A$ is a sequence $0=J_{0} \subsetneq$ $J_{1} \subsetneq \cdots \subsetneq J_{n}=A$ of stratifying ideals of $A$ such that each $J_{i} / J_{i-1}$ is a projective $A / J_{i-1}$-module. If $A$-mod has a strict stratifying system with quasi-poset $\Lambda$, then it has a standard stratification of length $n=|\bar{\Lambda}|$; see [Du et al. 1998, Theorem 1.2.8].

In the case of a finite dimensional algebra $A$ over a field $k$, the notion of a strict stratifying system $\{\Delta(\lambda)\}_{\lambda \in \Lambda}$ for $A$-mod simplifies somewhat. In this case, it can be assumed that each $\Delta(\lambda)$ has an irreducible head $L(\lambda)$, that $\lambda \neq \mu \Longrightarrow L(\lambda) \varsubsetneqq L(\mu)$, and that $P(\lambda)$ is indecomposable. Two caveats are in order, however: (i) it may be necessary to enlarge the base set $\Lambda$ to be able to index all the irreducible modules, though $\bar{\Lambda}$ can remain the same; (ii) it may be easier to verify (1), (2), and (3) over a larger ring and then base change. The irreducible head versions of the $\Delta(\lambda)$ can then be obtained as direct summands of the base-changed versions.

When the algebra $A$ arises as an endomorphism algebra $A=\operatorname{End}_{B}(T)$, there is a useful theory for obtaining a strict stratifying system for $A$-mod. In fact, this is how such stratifying systems initially arose; see [Cline et al. 1996; Du et al. 1998]. This approach is followed in the proof of the main theorem in this paper. For convenience, we summarize the sufficient conditions that will be used, all taken from [Du et al. 1998, Theorem 1.2.10]. 
Theorem 1.1. Let $B$ be a finitely generated projective $R$-algebra and let $T$ be a finitely generated right $B$-module which is projective over $R$. Define $A:=\operatorname{End}_{B}(T)$. Assume that $T=\oplus_{\lambda \in \Lambda} T_{\lambda}$, where $\Lambda$ is a finite quasi-poset. For $\lambda \in \Lambda$, assume there is given a fixed $R$-submodule $S_{\lambda} \subseteq T_{\lambda}$ and an increasing filtration $F_{\lambda}^{\bullet}: 0=F_{\lambda}^{0} \subseteq$ $F_{\lambda}^{1} \subseteq \cdots \subseteq F_{\lambda}^{t(\lambda)}=T_{\lambda}$ satisfying the following conditions:

(1) For $\lambda \in \Lambda, F_{\lambda}^{\bullet}$ has bottom section $F_{\lambda}^{1} / F_{\lambda}^{0} \cong S_{\lambda}$ and higher sections $F_{\lambda}^{i+1} / F_{\lambda}^{i}$ $(1 \leq i \leq t(\lambda)-1)$ of the form $S_{v}$ with $\bar{v}>\bar{\lambda}$.

(2) For $\lambda, \mu \in \Lambda, \operatorname{Hom}_{B}\left(S_{\mu}, T_{\lambda}\right) \neq 0 \Rightarrow \lambda \leq \mu$.

(3) For $\lambda \in \Lambda, \operatorname{Ext}_{B}^{1}\left(T_{\lambda} / F_{\lambda}^{i}, T\right)=0$ for all $i$.

Let $A=\operatorname{End}_{B}(T)$ and, for $\lambda \in \Lambda$, define $\Delta(\lambda):=\operatorname{Hom}_{B}\left(S_{\lambda}, T\right) \in A$-mod. Assume that each $\Delta(\lambda)$ is $R$-projective. Then $\{\Delta(\lambda)\}_{\lambda \in \Lambda}$ is a strict stratifying system for A-mod.

It is interesting to note that these sufficient conditions are not, in general, preserved under base change, though the resulting strict stratifying systems are preserved (becoming strict stratifying systems for the base-changed version of the algebra $A$ ).

1B. Cells and q-permutation modules. We assume familiarity with KazhdanLusztig cell theory for the Coxeter systems $(W, S)$. See, for instance, [Deng et al. 2008; Lusztig 2003]. In Conjecture 1.2 below and in Theorem 5.6, the set $\Lambda$ will be the set $\Omega$ of left Kazhdan-Lusztig cells for $(W, S)$. For each $\omega \in \Omega$, let

$$
S(\omega):=\mathcal{H}^{{ }_{L} \omega} / \mathcal{H}^{<{ }_{L} \omega} \in \mathcal{H}-\bmod
$$

be the corresponding left cell module. It is known that $S(\omega)$ is a free $\mathcal{Z}$-module with basis corresponding to certain Kazhdan-Lusztig basis elements $C_{x}^{\prime}, x \in \omega$; see Section 2. The corresponding dual left cell module is defined

$$
S_{\omega}:=\operatorname{Hom}_{\mathcal{Z}}(S(\omega), \mathcal{Z}) \in \bmod -\mathcal{H} .
$$

It is regarded as a right $\mathcal{H}$-module. Because $S(\omega)$ and hence $S_{\omega}$ are free over $\mathcal{Z}$, if $R$ is a commutative $\mathcal{Z}$-module, we can define

$$
\left\{\begin{array}{l}
S_{R}(\omega):=S(\omega) \otimes_{\mathcal{Z}} R \\
S_{\omega, R}:=S_{\omega} \otimes_{\mathcal{Z}} R=\operatorname{Hom}_{R}\left(S_{R}(\omega), R\right) .
\end{array}\right.
$$

For the special choice $R=\mathscr{Q}$ - see (1C.1) below for the definition of $\mathscr{Q}$ - we also use the notations

$$
\left\{\begin{array}{l}
\tilde{S}(\omega):=S_{\mathscr{Q}}(\omega), \\
\tilde{S}_{\omega}:=S_{\omega, \mathscr{Q}}, \quad \omega \in \Omega .
\end{array}\right.
$$

In addition, for $\lambda \subseteq S$, let $W_{\lambda}$ be the parabolic subgroup of $W$ generated by the $s \in \lambda$, and put $x_{\lambda}=\sum_{w \in W_{\lambda}} T_{w}$, with $T_{w}$ as in (1.1) above. The induced modules 
$x_{\lambda} \mathcal{H}$ (also called $q$-permutation modules) have an increasing filtration with sections $S_{\omega}$ for various $\omega \in \Omega$ (precisely, those left cells $\omega$ whose right set $\mathscr{R}(\omega)$ contains $\lambda)$.

Let $\mathcal{T}=\bigoplus_{\lambda} x_{\lambda} \mathcal{H}$, and $\mathcal{A}:=\operatorname{End}_{\mathcal{H}}(\mathcal{T})$. For $\omega \in \Omega$, put $\Delta(\omega):=\operatorname{Hom}_{\mathcal{H}}\left(S_{\omega}, \mathcal{T}\right) \in$ $\mathcal{A}$-mod. The algebra $\mathcal{A}$ is very well behaved in type A, a $q$-Schur algebra; a theme of [Du et al. 1998] was that suitable enlargements, appropriately compatible with two-sided cell theory, should have similar good properties for all types.

Each two-sided cell may be identified with the set of left cells it contains, and the resulting collection $\bar{\Omega}$ of sets of left cells is a partition of $\Omega$. There are various natural preorders on $\Omega$, but we will be mainly interested in those whose associated equivalence relation has precisely the set $\bar{\Omega}$ as its associated partition. We call such a preorder strictly compatible with $\bar{\Omega}$.

1C. A conjecture. Now we are ready to state the following conjecture, which is a variation (see the Appendix) on [op. cit., Conjecture 2.5.2]. We informally think of the algebra $\mathcal{A}^{+}$in the conjecture as an extension of $\mathcal{A}$ as a Hecke endomorphism algebra (justifying the title of the paper).

Conjecture 1.2. There exists a preorder $\leq$ on the set $\Omega$ of left cells in $W$, strictly compatible with its partition $\bar{\Omega}$ into two-sided cells, and a right $\mathcal{H}$-module $\mathcal{X}$ such that the following statements hold:

(1) $\mathcal{X}$ has an finite filtration with sections of the form $S_{\omega}, \omega \in \Omega$.

(2) Let $\mathcal{T}^{+}:=\mathcal{T} \oplus \mathcal{X}$ and put

$$
\left\{\begin{array}{l}
\mathcal{A}^{+}:=\operatorname{End}_{\mathcal{H}}\left(\mathcal{T}^{+}\right), \\
\Delta^{+}(\omega):=\operatorname{Hom}_{\mathcal{H}}\left(S_{\omega}, \mathcal{T}^{+}\right), \quad \text { for any } \omega \in \Omega .
\end{array}\right.
$$

Then, for any commutative, Noetherian $\mathcal{Z}$-algebra $R$, the set $\left\{\Delta^{+}(\omega)_{R}\right\}_{\omega \in \Omega}$ is a strict stratifying system for $\mathcal{A}_{R}^{+}$-mod relative to the quasi-poset $(\Omega, \leq)$.

The main result of this paper, given in Theorem 5.6, establishes a special "local case" of this conjecture. A more detailed description of this theorem requires some preliminary notation. Throughout this paper, $e$ is positive integer $(\neq 2$ in our main results). Let $\Phi_{2 e}(t)$ denote the (cyclotomic) minimum polynomial for a primitive $2 e$ th root of unity $\sqrt{\zeta}=\exp (2 \pi i / 2 e) \in \mathbb{C}$. Fix a modular system $(K, \mathscr{Q}, k)$ by letting

$$
\begin{cases}\mathscr{Q}:=\mathbb{Q}\left[t, t^{-1]}\right]_{\mathfrak{p}}, & \text { where } \mathfrak{p}=\left(\Phi_{2 e}(t)\right) ; \\ K:=\mathbb{Q}(t), & \text { the fraction field of } \mathscr{Q} ; \\ k:=\mathscr{Q} / \mathfrak{m} \cong \mathbb{Q}(\sqrt{\zeta}), & \text { the residue field of } \mathscr{Q} .\end{cases}
$$

Here $\mathfrak{m}$ denotes the maximal ideal of the DVR $\mathscr{Q}$. With some abuse of notation, we sometimes identify $\sqrt{\zeta}$ with its image in $k$. (Without passing to an extension 
or completion, the ring $\mathscr{Q}$ might not have such a root of unity in it.) The algebra $\mathcal{H}_{\mathbb{Q}(t)}$ is split semisimple, with irreducible modules corresponding to the irreducible modules of the group algebra $\mathbb{Q} W$. The $\mathscr{Q}$-algebra

$$
\widetilde{\mathcal{H}}:=\mathcal{H} \otimes_{\mathcal{Z}} \mathscr{Q}
$$

has a presentation by elements $T_{w} \otimes 1$ (which will still be denoted $T_{w}, w \in W$ ) completely analogous to (1.1). Similar remarks apply to $\mathcal{H}_{k}$, replacing $t^{2}$ by $\zeta$. Then Theorem 5.6 establishes that there exists an $\widetilde{\mathcal{H}}$-module $\widetilde{\mathcal{X}}$ which is filtered by dual left cell modules $\tilde{S}_{\omega}$ such that the analogues of conditions (1) and (2) over $\mathscr{Q}$ in Conjecture 1.2 hold. The preorder used in Theorem 5.6 is constructed as in [Ginzburg et al. 2003] from a "sorting function" $f$, and is discussed in detail in the next section.

With more work, it can be shown, when $e \neq 2$, that the $\mathscr{Q}$-algebra $\tilde{\mathcal{A}}^{+}:=\mathcal{A}_{\mathscr{Q}}^{+}$ is quasi-hereditary. This is done in Theorem 6.4. Then Theorem 6.5 identifies the module category for a base-changed version of this algebra with a RDAHA-category $\mathcal{O}$ in [Ginzburg et al. 2003]. Such an identification in type $A$ was conjectured in [loc. cit.], and then proved by Rouquier in [2008] (when $e \neq 2$ ).

Generally speaking, this paper focuses on the "equal parameter" case, i.e., all $c_{s}=1$ in (1.1), which covers the Hecke algebras relevant to all untwisted finite Chevalley groups. We will assume this condition unless explicitly stated otherwise, avoiding a number of complications involving Kazhdan-Lusztig basis elements and Lusztig's algebra $\mathcal{J}$. In this context, the critical Proposition 3.1 depends on results of [Ginzburg et al. 2003] which, in part, were only determined in the equal parameter case. Nevertheless, much of our discussion applies in the unequal parameter cases. In particular, we mention that the elementary, but important, Lemma 4.3 is stated and proved using unequal parameter notation. This encourages the authors to believe the main results are also provable in the unequal parameter case, though this has not yet been carried out. Note that all the rank 2 cases are treated in [Du et al. 1998], leaving the quasisplit cases with rank $>2$. All these quasisplit cases have parameters confined to the set $\{1,2,3\}$.

\section{Some preliminaries}

This section recalls some mostly well-known facts and fixes notation regarding cell theory. Let $W$ be a finite Weyl group associated to a finite root system $\Phi$ with a fixed set of simple roots $\Pi$. Let $S:=\left\{s_{\alpha} \mid \alpha \in \Pi\right\}$. Let $\mathcal{H}$ be a Hecke algebra over $\mathcal{Z}$ defined by (1.1). We assume (unless explicitly noted otherwise) that each $c_{s}=1$ for $s \in S$. Thus, $(W, S)$ corresponds, in the language of the introduction, to some types of split Chevalley groups, though we will have no further need of that context. 
Let

$$
C_{w}^{\prime}=t^{-l(w)} \sum_{y \leq w} P_{y, w} T_{y},
$$

where the $P_{y, w}$ is a Kazhdan-Lusztig polynomial in $\mathfrak{q}:=t^{2}$. Then $\left\{C_{w}^{\prime}\right\}_{w \in W}$ is a Kazhdan-Lusztig (or canonical) basis for $\mathcal{H}$. The element $C_{x}^{\prime}$ is denoted $c_{x}$ in [Lusztig 2003], a reference we frequently quote. Let $h_{x, y, z} \in \mathcal{Z}$ denote the structure constants. In other words,

$$
C_{x}^{\prime} C_{y}^{\prime}=\sum_{z \in W} h_{x, y, z} C_{z}^{\prime}
$$

Using the preorders $\leq_{L}$ and $\leq_{R}$ on $W$, the positivity (see [Deng et al. 2008, §7.8]) of the coefficients of the $h_{x, y, z}$ implies

$$
h_{x, y, z} \neq 0 \Rightarrow z \leq_{L} y \text { and } z \leq_{R} x \text {. }
$$

The Lusztig function $a: W \rightarrow \mathbb{N}$ is defined as follows. For $z \in W$, let $a(z)$ be the smallest nonnegative integer such that $t^{a(z)} h_{x, y, z} \in \mathbb{N}[t]$ for all $x, y \in W$. It may equally be defined as the smallest nonnegative integer such that $t^{-a(x)} h_{x, y, z} \in \mathbb{N}\left[t^{-1}\right]$, as used in [Lusztig 2003] (or see [Deng et al. 2008, §7.8]). In fact, each $h_{x, y, z}$ is invariant under the automorphism $\mathcal{Z} \rightarrow \mathcal{Z}$ sending $t$ to $t^{-1}$. It is not difficult to see that $a(z)=a\left(z^{-1}\right)$. For $x, y, z \in W$, let $\gamma_{x, y, z}$ be the coefficient of $t^{-a(z)}$ in $h_{x, y, z^{-1}}$. Also, by [Lusztig 2003, Conjectures 14.2(P8) and 15.6],

$$
\gamma_{x, y, z} \neq 0 \Rightarrow x \sim_{L} y^{-1}, y \sim_{L} z^{-1}, z \sim_{L} x^{-1} .
$$

The function $a$ is constant on two-sided cells in $W$, and so can be regarded as a function with values in $\mathbb{N}$ on (a) the set of two-sided cells; (b) the set of left (or right) cells; and (c) the set $\operatorname{Irr}(\mathbb{Q} W)$ of irreducible $\mathbb{Q} W$-modules. ${ }^{1}$ In addition, $a$ is related to the generic degrees $d_{E}, E \in \operatorname{Irr}(\mathbb{Q} W)$. For $E \in \operatorname{Irr}(\mathbb{Q} W)$, let $d_{E}=b t^{a_{E}}+\cdots+c t^{A_{E}}$, with $a_{E} \leq A_{E}$ and $b c \neq 0$, so that $t^{a_{E}}$ and $t^{A_{E}}$ are the smallest and largest powers of $t$ appearing nontrivially in $d_{E}$, respectively. Then $a_{E}=a(E)$; see [Lusztig 2003, Proposition 20.6]. Also, as noted in [Ginzburg et al. 2003, §6], $A_{E}=N-a(E \otimes \mathrm{det})$, where $N$ is the number of positive roots in $\Phi$. Following [Ginzburg et al. 2003, §6], we will use the "sorting function" $f: \operatorname{Irr}(\mathbb{Q} W) \rightarrow \mathbb{N}$ defined by

$$
f(E)=a_{E}+A_{E}=a(E)+N-a(E \otimes \mathrm{det}) .
$$

The function $f$ is also constant on two-sided cells: if $E$ is an irreducible $\mathbb{Q} W$ module associated to a two-sided cell $\boldsymbol{c}$, then $E \otimes$ det is an irreducible module associated to the two-sided cell $w_{0} \boldsymbol{c}$. See [Lusztig 1984, Lemma 5.14(iii)].

The function $f$ is used in [Ginzburg et al. 2003] to define various order structures on the set $\operatorname{Irr}(\mathbb{Q} W)$ of irreducible $\mathbb{Q} W$-modules. Put $E<_{f} E^{\prime}$ (our notation) provided

\footnotetext{
${ }^{1}$ It is well known that $\mathbb{Q}$ is a splitting field for $W$ [Benard 1971].
} 
$f(E)<f\left(E^{\prime}\right)$. There are at least two natural ways to extend $<_{f}$ to a preorder. The first way, which is only in the background for us, is to set $E \preceq_{f} E^{\prime} \Longleftrightarrow E \cong E^{\prime}$ or $E<_{f} E^{\prime}$. This gives a poset structure, and is used, in effect, by [Ginzburg et al. 2003] for defining a highest weight category $\mathcal{O}$; see [op. cit., §2.5, §6.2.1].

We use $<_{f}$ here to define a preorder $\leq_{f}$ on the set $\Omega$ of left cells: First, observe that the function $f$ above is constant on irreducible modules associated to the same left cell (or even the same two-sided cell) and so may be viewed as a function on $\Omega$. We can now define the (somewhat subtle) preorder $\leq_{f}$ on $\Omega$ by setting $\omega \leq_{f} \omega^{\prime}$ (for $\omega, \omega^{\prime} \in \Omega$ ) if and only if either $f(\omega)<f\left(\omega^{\prime}\right)$, or $\omega$ and $\omega^{\prime}$ lie in the same two-sided cell. Note that the "equivalence classes" of the preorder $\leq_{f}$ identify with the set of two-sided cells - thus, $\leq_{f}$ is strictly compatible with the set of two-sided cells in the sense of Section 1. Also,

$$
E<_{L R} E^{\prime} \Rightarrow E^{\prime}<_{f} E
$$

see [op. cit., Lemma 6.6]. Here $E, E^{\prime}$ are in $\operatorname{Irr}(\mathbb{Q} W)$, and the notation $E<_{L R} E^{\prime}$ means that the two-sided cell associated with $E$ is strictly smaller than that associated with $E^{\prime}$, with respect to the Kazhdan-Lusztig order on two-sided cells. A property similar to (2.6) holds if $<_{L R}$ is replaced with $<_{L}$, defined similarly, but using left cells. In terms of $\Omega$, this left cell version reads:

$$
\omega, \omega^{\prime} \in \Omega, \omega<_{L} \omega^{\prime} \Rightarrow f(\omega)>f\left(\omega^{\prime}\right) .
$$

Notice that (2.7) follows from (2.6) using [Lusztig 1987a, Corollary 1.9(c)]. (The latter result implies that $\omega, \omega^{\prime}$ on the left in (2.7) cannot belong to the same twosided cell.) Thus, the preorder $\leq_{f}$ is a refinement of the preorder $\leq_{L}^{\text {op }}$ on $\Omega$, and $\leq_{f}$ induces on the set of two-sided cells a refinement of the partial order $\leq_{L R}^{\mathrm{op}}$. For further discussion, see the Appendix.

\section{3. (Dual) Specht modules of Ginzburg-Guay-Opdam-Rouquier}

The asymptotic form $\mathcal{J}$ of $\mathcal{H}$ is a ring with $\mathbb{Z}$-basis $\left\{j_{x} \mid x \in W\right\}$ and multiplication

$$
j_{x} j_{y}=\sum_{z} \gamma_{x, y, z^{-1}} j_{z}
$$

This ring was originally introduced in [Lusztig 1987a], though we follow [Lusztig 2003, §18.3], using a slightly different notation.

3A. The mapping $\varpi$ and its properties. As per [op. cit., §18.9], define a $\mathcal{Z}$-algebra homomorphism

$$
\varpi: \mathcal{H} \rightarrow \mathcal{J}_{\mathcal{Z}}=\mathcal{J} \otimes \mathcal{Z}, \quad C_{w}^{\prime} \mapsto \sum_{z \in W} \sum_{\substack{d \in \boldsymbol{D} \\ a(d)=a(z)}} h_{w, d, z} j_{z},
$$


where $\boldsymbol{D}$ is the set of distinguished involutions in $W$. Also, for any $\mathcal{Z}$-algebra $R$, there is an algebra homomorphism $\varpi_{R}: \mathcal{H}_{R}=\mathcal{H} \otimes_{\mathcal{Z}} R \rightarrow \mathcal{J}_{R}=\mathcal{J}_{\mathcal{Z}} \otimes_{\mathcal{Z}} R$, obtained by base change. In obvious cases, we often drop the subscript $R$ from $\varpi_{R}$.

In particular, $\varpi_{\mathbb{Q}(t)}$ becomes an isomorphism

$$
\varpi=\varpi_{\mathbb{Q}(t)}: \mathcal{H}_{\mathbb{Q}(t)} \stackrel{\sim}{\rightarrow} \mathcal{J}_{\mathbb{Q}(t)} .
$$

See [Lusztig 1987a]. Also, $\varpi$ induces a monomorphism

$$
\varpi=\varpi_{\mathbb{Q}\left[t, t^{-1}\right]}: \mathcal{H}_{\mathbb{Q}\left[t, t^{-1}\right]} \hookrightarrow \mathcal{J}_{\mathbb{Q}\left[t, t^{-1}\right]}=\mathcal{J}_{\mathbb{Q}} \otimes \mathbb{Q}\left[t, t^{-1}\right] .
$$

Moreover, base change to $\mathbb{Q}\left[t, t^{-1}\right] /(t-1)$ induces an isomorphism

$$
\varpi=\varpi_{\mathbb{Q}}: \mathbb{Q} W \stackrel{\sim}{\longrightarrow} \mathcal{J}_{\mathbb{Q}}
$$

(compare [Lusztig 1987b, Proposition 1.7]). This allows us to identify irreducible $\mathbb{Q} W$-modules with irreducible $\mathcal{J}_{\mathbb{Q}}$-modules. ${ }^{2}$

For the irreducible (left) $\mathcal{J}_{\mathbb{Q}}$-module identified with $E \in \operatorname{Irr}(\mathbb{Q} W)$, the (left) $\mathcal{H}_{\mathbb{Q}\left[t, t^{-1}\right]^{-m o d u l e}}$

$$
S(E):=\varpi^{*}\left(E \otimes \mathbb{Q}\left[t, t^{-1}\right]\right)=\varpi^{*}\left(E_{\mathbb{Q}\left[t, t^{-1}\right]}\right)
$$

is called here a dual Specht module for $\mathcal{H}_{\mathbb{Q}\left[t, t^{-1}\right]}$; compare [Ginzburg et al. 2003, Corollary 6.10]. ${ }^{3}$ Note that $S(E) \cong E_{\mathbb{Q}\left[t, t^{-1}\right]}$ as a $\mathbb{Q}\left[t, t^{-1}\right]$-module. Therefore, $S(E)$ is a free $\mathbb{Q}\left[t, t^{-1}\right]$-module. Putting $S_{E}=\operatorname{Hom}_{\mathbb{Q}\left[t, t^{-1}\right]}\left(S(E), \mathbb{Q}\left[t, t^{-1}\right]\right)$, define

$$
\left\{\begin{array}{l}
\tilde{S}(E):=S_{\mathscr{Q}}(E) \\
\tilde{S}_{E}:=S_{E, \mathscr{Q}}
\end{array}\right.
$$

where, in general, for base change to a commutative, Noetherian $\mathbb{Q}\left[t, t^{-1}\right]$-algebra $R$,

$$
\left\{\begin{array}{l}
S_{R}(E):=S(E) \otimes_{\mathbb{Q}\left[t, t^{-1}\right]} R, \\
S_{E, R}:=S_{E} \otimes_{\mathbb{Q}\left[t, t^{-1}\right]} R \cong \operatorname{Hom}_{R}\left(S_{R}(E), R\right) .
\end{array}\right.
$$

\footnotetext{
${ }^{2}$ The map $\varpi$ is the composition $\phi \circ \dagger$, where $\phi$ and $\dagger$ are defined in [Lusztig 2003, §18.9] and [op. cit., §3.5], respectively. The numbers $\hat{n}_{z}$ appearing there (which are \pm 1 by definition in [op. cit., $\S 18.8$ ]) are all equal to 1 , because of the positivity (see [op. cit., §7.8]) of the structure constants appearing in [op. cit., 14.1]. This $\varpi$ is not the same one as defined in [Ginzburg et al. 2003, p. 647], where the $C$-basis was used. Nevertheless, the arguments of [op. cit., §6] go through, using the $C^{\prime}$-basis and our $\varpi$ (see Remark 5.2 below), so [op. cit., Theorem 6.8] guarantees the modules $S_{\mathbb{C}}(E)$ defined below using our setup are the same, at least up to a (two-sided cell preserving) permutation of the isomorphism types labeled by the $E$, as the modules $S(E)$ defined in [op. cit., Definition 6.1] with $R=\mathbb{C}$. The proof of [op. cit., Theorem 6.8] also establishes such an identification of the various modules $S_{R}(E)$ when $R$ is a completion of $\mathbb{C}\left[t, t^{-1}\right]$.

${ }^{3}$ In [Ginzburg et al. 2003, Definition 6.1], the module $S(E)$ there is called a standard module. Our choice of terminology is justified by the discussion following the proof of Lemma 5.1 below.
} 
The following proposition is proved using RDAHAs, and it is the only ingredient in the proof of Theorem 5.6 where these algebras are used.

Proposition 3.1. Assume that $e \neq 2$. Suppose $E, E^{\prime}$ are irreducible $\mathbb{Q} W$-modules. If $E \not E^{\prime}$ and

$$
\operatorname{Hom}_{\mathcal{H}_{k}}\left(S_{k}(E), S_{k}\left(E^{\prime}\right)\right) \neq 0,
$$

then $f(E)<f\left(E^{\prime}\right)$. Also, $\operatorname{Hom}_{\mathcal{H}_{k}}\left(S_{k}(E), S_{k}(E)\right) \cong k$.

Proof. Without loss, we replace $k$ in the statement of the proposition by $\mathbb{C}$, using the analogous definitions of $S_{\mathbb{C}}(E)$. In addition, the statement of the proposition is invariant under any two-sided cell preserving permutation of the labeling of the irreducible modules. After applying such a permutation on the right (say) we may assume, by [Ginzburg et al. 2003, Theorem 6.8] and taking into account note 1 on page 235 , that

$$
\mathrm{KZ}(\Delta(E)) \cong S_{\mathbb{C}}(E),
$$

where

(1) $\Delta(E)$ is the standard module for a highest weight category $\mathcal{O}$ given in [op. cit.], having partial order $\leq_{f}$ (see [op. cit., Lemma 2.9, §6.2.1]) on its set of isomorphism classes of irreducible modules, which are indexed by isomorphism classes of irreducible $\mathbb{Q} W$-modules. We take $k_{H, 1}=1 / e>0$ in [op. cit.] above Theorem 6.8 and in Remark 3.2 there.

(2) The functor $\mathrm{KZ}: \mathcal{O} \rightarrow \overline{\mathcal{O}}$ is naturally isomorphic to the quotient map $M \mapsto \bar{M}$ in [op. cit., Proposition 5.9 and Theorem 5.14], the quotient category there identifying with $\mathcal{H}_{\mathbb{C}}$-mod.

Using [Ginzburg et al. 2003, Proposition 5.9], which requires $e \neq 2$, we have, for any irreducible $\mathbb{C} W$-modules $E$ and $E^{\prime}$,

$$
\operatorname{Hom}_{\mathcal{O}}\left(\Delta(E), \Delta\left(E^{\prime}\right)\right) \cong \operatorname{Hom}_{\overline{\mathcal{O}}}\left(\bar{\Delta}(E), \bar{\Delta}\left(E^{\prime}\right)\right) \cong \operatorname{Hom}_{\mathcal{H}_{\mathbb{C}}}\left(S_{\mathbb{C}}(E), S_{\mathbb{C}}\left(E^{\prime}\right)\right) .
$$

If $E \nsucceq E^{\prime}$, then $\Delta(E) \nsucceq \Delta\left(E^{\prime}\right)$ and $\operatorname{Hom}_{\mathcal{O}}\left(\Delta(E), \Delta\left(E^{\prime}\right)\right) \neq 0$ implies that $E<_{f} E^{\prime}$, i.e., $f(E)<f\left(E^{\prime}\right)$.

On the other hand, if $E \cong E^{\prime}$, then $\operatorname{Hom}_{\mathcal{O}}\left(\Delta(E), \Delta\left(E^{\prime}\right)\right) \cong \mathbb{C}$. This implies

$$
\operatorname{Hom}_{\mathcal{H}}\left(S_{\mathbb{C}}(E), S_{\mathbb{C}}\left(E^{\prime}\right)\right) \cong \mathbb{C} .
$$

Returning to the original $k=\mathbb{Q}(\sqrt{\zeta})$, we may conclude the same isomorphism holds in the original setting as well.

Corollary 3.2. Assume $e \neq 2$. Let $E, E^{\prime}$ be irreducible $\mathbb{Q} W$-modules. Then

$$
\operatorname{Ext}_{\tilde{\mathcal{H}}}^{1}\left(\tilde{S}(E), \tilde{S}\left(E^{\prime}\right)\right) \neq 0 \Rightarrow f(E)<f\left(E^{\prime}\right) .
$$

In particular, $\operatorname{Ext}_{\tilde{\mathcal{H}}}^{1}(\tilde{S}(E), \tilde{S}(E))=0$. 
Proof. In (1C.1) let $\pi=\Phi_{2 e}(t)$ be the generator of the maximal ideal $\mathfrak{m}$ of $\mathscr{Q}$, and consider the short exact sequence

$$
0 \longrightarrow \tilde{S}\left(E^{\prime}\right) \stackrel{\pi}{\longrightarrow} \tilde{S}\left(E^{\prime}\right) \longrightarrow S_{k}\left(E^{\prime}\right) \longrightarrow 0 .
$$

By the long exact sequence of Ext, there is an exact sequence

$$
\begin{aligned}
0 \rightarrow \operatorname{Hom}_{\tilde{\mathcal{H}}}\left(\tilde{S}(E), \tilde{S}\left(E^{\prime}\right)\right) & \stackrel{\pi}{\longrightarrow} \operatorname{Hom}_{\tilde{\mathcal{H}}}\left(\tilde{S}(E), \tilde{S}\left(E^{\prime}\right)\right) \longrightarrow \operatorname{Hom}_{\tilde{\mathcal{H}}_{k}}\left(S_{k}(E), S_{k}\left(E^{\prime}\right)\right) \\
& \longrightarrow \operatorname{Ext}_{\tilde{\mathcal{H}}}^{1}\left(\tilde{S}(E), \tilde{S}\left(E^{\prime}\right)\right) \stackrel{\pi}{\longrightarrow} \operatorname{Ext}_{\tilde{\mathcal{H}}}^{1}\left(\tilde{S}(E), \tilde{S}\left(E^{\prime}\right)\right) \\
& \longrightarrow \operatorname{Ext}_{\tilde{\mathcal{H}}_{k}}^{1}\left(S_{k}(E), S_{k}\left(E^{\prime}\right)\right) .
\end{aligned}
$$

Because $\mathcal{H}_{\mathbb{Q}(t)}=\tilde{\mathcal{H}}_{\mathbb{Q}(t)}$ is semisimple,

$$
\operatorname{Ext}_{\tilde{\mathcal{H}}}^{1}\left(\tilde{S}(E), \tilde{S}\left(E^{\prime}\right)\right)_{\mathbb{Q}(t)} \cong \operatorname{Ext}_{\mathcal{H}_{\mathbb{Q}(t)}}^{1}\left(S(E)_{\mathbb{Q}(t)}, S\left(E^{\prime}\right)_{\mathbb{Q}(t)}\right)=0 .
$$

In other words, if it is nonzero, $\operatorname{Ext}_{\tilde{\mathcal{H}}}^{1}\left(\tilde{S}(E), \tilde{S}\left(E^{\prime}\right)\right)$ is a torsion module, so the map

$$
\operatorname{Ext}_{\tilde{\mathcal{H}}}^{1}\left(\tilde{S}(E), \tilde{S}\left(E^{\prime}\right)\right) \stackrel{\pi}{\longrightarrow} \operatorname{Ext}_{\tilde{\mathcal{H}}}^{1}\left(\tilde{S}(E), \tilde{S}\left(E^{\prime}\right)\right)
$$

is not injective. Thus, it suffices to prove that when $f(E) \neq f\left(E^{\prime}\right)$, the map

$$
\operatorname{Hom}_{\tilde{\mathcal{H}}}\left(\tilde{S}(E), \tilde{S}\left(E^{\prime}\right)\right) \longrightarrow \operatorname{Hom}_{\tilde{\mathcal{H}}_{k}}\left(S_{k}(E), S_{k}\left(E^{\prime}\right)\right)
$$

is surjective. If $E \nsubseteq E^{\prime}$, Proposition 3.1 gives $\operatorname{Hom}_{\tilde{\mathcal{H}}_{k}}\left(S_{k}(E), S_{k}\left(E^{\prime}\right)\right)=0$ implying the surjectivity of (3A.6) trivially. On the other hand, if $E \cong E^{\prime}$, the proposition gives $\operatorname{Hom}_{\mathcal{H}_{k}}\left(S_{k}(E), S_{k}\left(E^{\prime}\right)\right) \cong k$. This also gives surjectivity of the map in (3A.6), since it becomes surjective upon restriction to $\mathscr{Q} \subseteq \operatorname{Hom}_{\tilde{\mathcal{H}}}\left(\tilde{S}(E), \tilde{S}\left(E^{\prime}\right)\right.$ ) (taking $\left.E^{\prime}=E\right)$.

\section{Two preliminary lemmas}

Let $R$ be a commutative ring and let $\mathscr{C}$ be an abelian $R$-category. For $A, B \in \mathscr{C}$, let $\operatorname{Ext}_{\mathscr{C}}^{1}(A, B)$ denote the Yoneda group of extensions of $A$ by $B$. (We do not require the higher Ext-groups in this section.) Let $M, Y \in \mathscr{C}$, and suppose that $\operatorname{Ext}_{\mathscr{C}}^{1}(M, Y)$ is generated as an $R$-module by elements $\epsilon_{1}, \ldots, \epsilon_{m}$. Let $\chi:=\oplus_{i} \epsilon_{i} \in \operatorname{Ext}_{\mathscr{C}}^{1}\left(M^{\oplus m}, Y\right)$ correspond to the short exact sequence $0 \rightarrow Y \rightarrow X \rightarrow M^{\oplus m} \rightarrow 0$.

Lemma 4.1. The map $\operatorname{Ext}_{\mathscr{C}}^{1}(M, Y) \longrightarrow \operatorname{Ext}_{\mathscr{C}}^{1}(M, X)$, induced by the inclusion $Y \hookrightarrow X$, is the zero map.

Proof. Using the "long" exact sequence of Ext", it suffices to show that the map $\delta$ in the sequence

$$
\operatorname{Hom}_{\mathscr{C}}(M, X) \longrightarrow \operatorname{Hom}_{\mathscr{C}}\left(M, M^{\oplus m}\right) \stackrel{\delta}{\longrightarrow} \operatorname{Ext}_{\mathscr{C}}^{1}(M, Y)
$$


is surjective - equivalently, that each $\epsilon_{i} \in \operatorname{Ext}_{\mathscr{C}}^{1}(M, Y)$ lies in the image of $\delta$. Let $0 \rightarrow Y \rightarrow X_{i} \rightarrow M \rightarrow 0$ correspond to $\epsilon_{i} \in \operatorname{Ext}_{\mathscr{C}}^{1}(M, Y)$. By construction, $\epsilon_{i}$ is the image of $\chi$ under the natural map

$$
j_{i}^{*}: \operatorname{Ext}_{\mathscr{C}}^{1}\left(M^{\oplus m}, Y\right) \longrightarrow \operatorname{Ext}_{\mathscr{C}}^{1}(M, Y),
$$

which is the pull-back of the inclusion $j_{i}$ of $M$ into the $i$-th summand of $M^{\oplus m}$. So there is a natural commutative diagram:

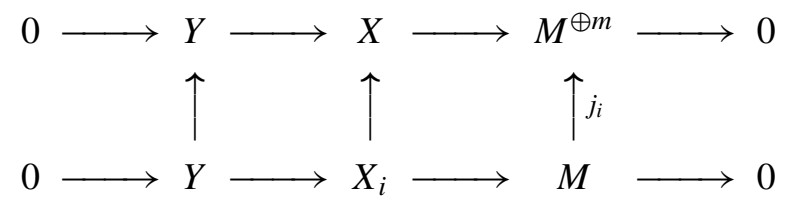

There is a corresponding commutative diagram

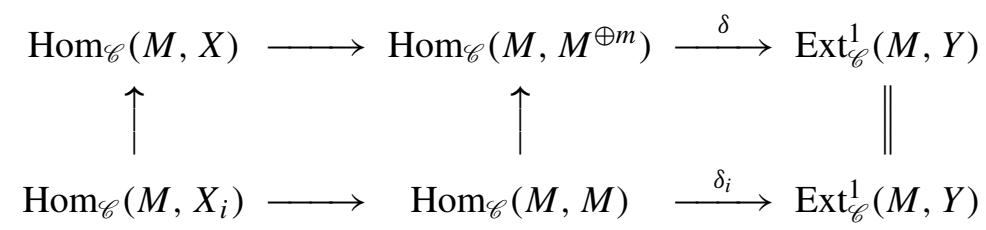

where each row is part of a "long" exact sequence. Then $\delta_{i}\left(1_{M}\right)=\epsilon_{i}$. Therefore, the commutativity of the right hand square in (4.7) immediately says that $\epsilon_{i}$ lies in the image of $\delta$.

This lemma together with the additivity of the functor Ext $_{\mathscr{C}}^{1}$ gives immediately the following.

Corollary 4.2. Maintain the setup above. If $\operatorname{Ext}_{\mathscr{C}}^{1}(M, M)=0$, then $\operatorname{Ext}_{\mathscr{C}}^{1}(M, X)=$ 0 .

Next, let $R$ be a commutative ring which is a $\mathcal{Z}$-algebra and write $q=t^{2} \cdot 1$, the image in $R$ of $t^{2} \in \mathcal{Z}$. We allow general parameters $c_{s}$ and $s \in S$ in (1.1) for the rest of this section.

Lemma 4.3. Let $\mathfrak{N} \subseteq \mathfrak{M}$ be left ideals in $\mathcal{H}_{R}$, with each spanned by the KazhdanLusztig basis elements $C_{y}^{\prime}$ that they contain. Let $s \in S$ be a simple reflection and assume either $\mathfrak{N}=0$ or that $q^{c_{s}}+1$ is not a zero divisor in $R$. Suppose $0 \neq x \in \mathfrak{M} / \mathfrak{N}$ satisfies

$$
T_{s} \cdot x=q^{c_{s}} x .
$$

Then $x$ is represented in $\mathfrak{M}$ by an R-linear combination of Kazhdan-Lusztig basis elements $C_{y}^{\prime}$ with sy $<y$.

Proof. Let $[m]$ denote the image in $\mathfrak{M} / \mathfrak{N}$ of $m \in \mathfrak{M}$. Note that $\mathfrak{M}$, $\mathfrak{N}$, and $\mathfrak{M} / \mathfrak{N}$ are all $R$-free, since the $C_{y}^{\prime}$ which belong to $\mathfrak{M}$ and $\mathfrak{N}$ form a basis for $\mathfrak{M}$ and $\mathfrak{N}$, 
respectively. The $R$-module $\mathfrak{M} / \mathfrak{N}$ has a basis consisting of all $\left[C_{y}^{\prime}\right] \neq 0$ with $C_{y}^{\prime} \in \mathfrak{M}$.

Write $x=\sum_{y} a_{y}\left[C_{y}^{\prime}\right]$ with $a_{y}\left[C_{y}^{\prime}\right] \neq 0$ and $C_{y}^{\prime} \in \mathfrak{M}$. Observe that, for $y \in W, s \in S$,

$$
s y<y \Longrightarrow T_{s} C_{y}^{\prime}=q^{c_{s}} C_{y}^{\prime} .
$$

Therefore, in the above expression for $x$, it may also be assumed that $s y>y$ for each nonzero term $a_{y}\left[C_{y}^{\prime}\right]$. Let $a_{w}\left[C_{w}^{\prime}\right] \neq 0$ be chosen with $w$ maximal among these $y$. In general, for $s y>y$, we have

$$
T_{s} C_{y}^{\prime}=-C_{y}^{\prime}+C_{s y}^{\prime}+\sum_{\substack{z<y \\ s z<z}} b_{z} C_{z}^{\prime}
$$

for various $b_{z} \in R$. Equating coefficients of $\left[C_{w}^{\prime}\right]$ gives by (4.8) that $\left(q^{c_{s}}+1\right) a_{w}=0$, since $C_{w}^{\prime}$ does not appear with any coefficient in the expressions $T_{s} C_{y}^{\prime}$ with $y \neq w$ and $s y>y$. Now the hypothesis on zero divisors forces $a_{w}=0$, a contradiction.

Remark 4.4. As observed in (4.9) above, elements $x \in \mathfrak{M} / \mathfrak{N}$ satisfying the conclusion of Lemma 4.3 also satisfy its hypothesis (4.8). Next, suppose that $\lambda \subseteq S$ and $\mathfrak{L}$ is any $\mathcal{H}_{R}$-module. By Frobenius reciprocity, the $R$-module $\operatorname{Hom}_{\mathcal{H}_{R}}\left(\mathcal{H}_{R} x_{\lambda}, \mathfrak{L}\right)$ identifies with the $R$-submodule $\mathcal{X} \subseteq \mathfrak{L}$ consisting of all $x \in \mathfrak{L}$ satisfying (4.8) for all $s \in \lambda$. Suppose $\mathfrak{L}$ can be realized as $\mathfrak{L}=\mathfrak{M} / \mathfrak{N}$, with $\mathfrak{M}, \mathfrak{N}$ as in the statement of Lemma 4.3. If $q^{c_{s}}+1$ is invertible in $R$ for all $s \in \lambda$, then the lemma implies that $\mathcal{X}$ has an $R$-basis consisting of all nonzero $\left[C_{y}^{\prime}\right]$ in $\mathfrak{L}$ with $s y<y$ for all $s \in \lambda$.

Thus, if $R^{\prime}$ is an $R$-algebra, then the $R^{\prime}$-module $\operatorname{Hom}_{\mathcal{H}_{R^{\prime}}}\left(\mathcal{H}_{R^{\prime}} x_{\lambda}, \mathfrak{L}_{R^{\prime}}\right)$ has essentially the "same basis." This fact will be used in proving the following corollary.

In the result below, we allow $c_{s} \neq 1$. In case $c_{s}=1$, assumption (2) is satisfied for $R=\mathscr{Q}$ if and only if $e \neq 2$.

Corollary 4.5. Suppose $R$ is a commutative domain with fraction field $F$, and assume that $R$ is also a $\mathcal{Z}$-algebra. Let $\lambda \subseteq S$. Assume that

(1) $\mathcal{H}_{F}$ is semisimple;

(2) $q^{c_{s}}+1$ is invertible in $R$, for each $s \in \lambda$.

Then, for any dual left cell module $S_{\omega, R}$ over $R$,

$$
\operatorname{Ext}_{\mathcal{H}_{R}}^{1}\left(S_{\omega, R}, x_{\lambda} \mathcal{H}_{R}\right)=0 .
$$

Proof. Put $\mathcal{S}:=S_{\omega, R}$. Using condition (1) and [Du et al. 1998, Lemma 1.2.13], it suffices to prove, for each $R^{\prime}=R /\langle d\rangle(d \in R)$, that the map

$$
\operatorname{Hom}_{\mathcal{H}_{R}}\left(\mathcal{S}, x_{\lambda} \mathcal{H}_{R}\right) \longrightarrow \operatorname{Hom}_{\mathcal{H}_{R^{\prime}}}\left(\mathcal{S}_{R^{\prime}}, x_{\lambda} \mathcal{H}_{R^{\prime}}\right)
$$

is surjective. Here $\mathcal{S}_{R^{\prime}}=\mathcal{S} \otimes_{R} R^{\prime}$. 
By [op. cit., Lemma 2.1.9], the left $\mathcal{H}_{R}$-module $\left(x_{\lambda} \mathcal{H}_{R}\right)^{*}:=\operatorname{Hom}_{R}\left(x_{\lambda} \mathcal{H}_{R}, R\right)$ is naturally isomorphic to $\mathcal{H}_{R} x_{\lambda}$. By hypothesis, $\mathcal{S}=\mathcal{L}^{*}$ is the dual of a left cell module $\mathcal{L}, R$-free by definition. Thus, $\mathcal{L} \cong \mathcal{S}^{*}$; also, $\left(\mathcal{H}_{R} x_{\lambda}\right)^{*} \cong x_{\lambda} \mathcal{H}_{R}$. There are similar isomorphisms for analogous $R^{\prime}$-modules (for which we use the same notation $\left.(-)^{*}\right)$. The functor $(-)^{*}$ provides a contravariant equivalence from the category of finitely generated $R$-free left $\mathcal{H}_{R}$-modules and the corresponding right $\mathcal{H}_{R}$-module category. A similar statement holds with $R$ replaced by $R^{\prime}$. Finally, there is a natural isomorphism $(-)^{*} \otimes_{R} R^{\prime} \stackrel{\sim}{\longrightarrow}\left(-\otimes_{R} R^{\prime}\right)^{*}$.

Consequently, it is sufficient to prove that

$$
\operatorname{Hom}_{\mathcal{H}_{R}}\left(\mathcal{H}_{R} x_{\lambda}, \mathcal{L}\right) \longrightarrow \operatorname{Hom}_{\mathcal{H}_{R^{\prime}}}\left(\mathcal{H}_{R^{\prime}} x_{\lambda}, \mathcal{L}_{R^{\prime}}\right)
$$

is surjective. (Here $\mathcal{L}_{R^{\prime}}$ denotes the left cell module in $\mathcal{H}_{R^{\prime}}$ defined by the same left cell as $\mathcal{L}$ for $\mathcal{H}$.) However, viewing $\mathcal{L}$ and $\mathcal{L}_{R^{\prime}}$ as cell modules (over $\mathcal{H}_{R}$ and $\mathcal{H}_{R^{\prime}}$, respectively), hypothesis (2), Lemma 4.3, and Remark 4.4 give the "same basis" (over $R$ and $R^{\prime}$, respectively).

\section{The construction of $\tilde{X}_{\omega}$ and the main theorem}

In this section, we prove the main result of the paper (Theorem 5.6).

Let $\mathscr{Q}$ be as in (1C.1). Recall that $\tilde{\mathcal{H}}$ denotes the $\mathscr{Q}$-algebra $\mathcal{H} \otimes_{\mathcal{Z}} \mathscr{Q}$. In general, modules for $\tilde{\mathcal{H}}$ are decorated with a "tilde" (e.g., $\tilde{X}$ ). In particular, we recall from (1B.3) the notations $\tilde{S}(\omega)$ and $\tilde{S}_{\omega}$.

5A. Preliminaries. Consider a left cell $\omega$ and let $\mathcal{J}_{\omega}=\sum_{y \in \omega} \mathbb{Z} j_{y}$. Then (2.4) implies that $\mathcal{J}_{\omega}$ is a left $\mathcal{J}$-module. Using the monomorphism $\varpi$ in Section 3, form the left $\mathcal{H}$-module $\varpi^{*}\left(\mathcal{J}_{\omega} \otimes \mathcal{Z}\right)$, the restriction of the $\mathcal{J}_{\mathcal{Z}}$-module $\mathcal{J}_{\omega} \otimes \mathcal{Z}$ to $\mathcal{H}$.

Lemma 5.1. There is an $\mathcal{H}$-module isomorphism

$$
\sigma: \varpi^{*}\left(\mathcal{J}_{\omega} \otimes \mathcal{Z}\right) \longrightarrow S(\omega):=\mathcal{H}^{{ }_{L}{ }^{\omega}} / \mathcal{H}^{<L} \omega
$$

induced by the map $\sigma: \mathcal{J}_{\mathcal{Z}} \rightarrow \mathcal{H}, j_{y} \mapsto C_{y}^{\prime}$. In particular, $\tilde{S}(\omega)$ is a direct sum of modules $\tilde{S}(E)$ for some $E \in \operatorname{Irr}(\mathbb{Q} W)$.

Proof. This is a refinement of [Lusztig 2003, §18.10]. We first observe that the map $\sigma$ clearly induces a $\mathcal{Z}$-module isomorphism. It remains to check for $y \in \omega$ that

$$
\sigma\left(\varpi\left(C_{x}^{\prime}\right) j_{y}\right) \equiv C_{x}^{\prime} C_{y}^{\prime} \quad \bmod \mathcal{H}^{<\omega}, \quad(x \in W)
$$

The proof of [op. cit, $\S 18.10(a)]^{4}$ gives the left hand equality in the expression

(5A.1) $\sigma\left(\varpi\left(C_{x}^{\prime}\right) j_{y}\right)=\sigma\left(\sum_{\substack{u \\ a(y)=a(u)}} h_{x, y, u} j_{u}\right)=\sum_{\substack{u \\ a(y)=a(u)}} h_{x, y, u} C_{u}^{\prime} \equiv C_{x}^{\prime} C_{y}^{\prime} \quad \bmod \mathcal{H}^{<L \omega}$.

\footnotetext{
${ }^{4}$ The main ingredient is [op. cit, §18.9(b)]. As previously noted, the $\hat{n}_{z}$ may be set equal to 1 .
} 
The middle equality is just the definition of $\sigma$. Finally, the right hand congruence follows from the fact that, when $h_{x, y, u} C_{u}^{\prime}$ is nonzero mod $\mathcal{H}^{{ }_{L} \omega}, u$ must belong to the same left cell $\omega$ as $y$, and hence have the same $a$-value.

If $W$ is of type $A$ and $\omega$ is the left cell containing the longest word $w_{0, \lambda}$ for a partition $\lambda$. Then $\varpi^{*}\left(\mathcal{J}_{\omega} \otimes \mathcal{Z}\right)$ is isomorphic to the left cell module whose dual is the Specht module $S_{\lambda}$. So $\tilde{S}(E)$ above could be called a "dual Specht module," with $\tilde{S}(E)^{*}$ a "Specht module." The modules $\tilde{S}_{\omega}$ are also candidates for the name "Specht module" [Du et al. 1998, p. 198].

Remark 5.2. A completely analogous result to Lemma 5.1 holds if the KazhdanLusztig $C$-basis (instead of the $C^{\prime}$-basis here) is used, as in [Ginzburg et al. 2003]. First, it follows from [Lusztig 1985, (3.2)] that the map (which we call $\tau$ ) $\mathcal{Z} \rightarrow \mathcal{Z}$, sending $t \mapsto-t$, takes the coefficients $h_{x, y, z}$ to analogous coefficients for the $C$-basis. Extend $\tau$ to an automorphism, still denoted $\tau$, of $\mathcal{J}_{\mathcal{Z}}$, taking $j_{x}$ to its $C$-analogue; we may put $\tau\left(j_{x}\right)=(-1)^{\ell(x)} j_{x}$. Thus, any expression $h_{x, y, z} j_{z}$ is sent to a $C$-basis analogue. In particular, $\varpi\left(C_{x}^{\prime}\right)$ is sent to $\varpi\left(C_{x}\right)$, where the latter $\varpi$ is taken in the $C$-basis set-up. Now it is clear from (5A.1) that the analogue of Lemma 5.1 holds in the $C$-basis set-up. Note the resulting left cell modules in $\mathcal{H}$ do not depend on which canonical basis is used. This allows an identification of the module $S(\omega)$ in Lemma 5.1 with its $C$-basis counterpart.

An analogous result holds for two-sided cells, e.g., the $\mathcal{H}$-module $\varpi^{*}\left(\mathcal{J}_{\underline{c}} \otimes_{\mathbb{Z}} \mathcal{Z}\right)$ in [Ginzburg et al. 2003, Corollary 6.4] does not depend on the whether the $C^{\prime}$-basis is used (as in this paper) or the $C$-basis is used (as in [op. cit]). We do not know, however, if the base-change of the automorphism $\tau$ to $\mathcal{J}_{\mathbb{Q}(t)}$ preserves the isomorphism types of irreducible $\mathcal{J}_{\mathbb{Q}(t)}$-modules, though their associated two-sided cells are preserved. This leads to the "permutation" language used in footnote 2 . In particular, we do not know if the bijection noted below [Ginzburg et al. 2003, Definition 6.1] depends on the choice of $C$ - or $C^{\prime}$-basis set-up, and could result in one choice leading to an identification which is a (two-sided cell preserving) permutation of the other.

Corollary 5.3. Assume that $e \neq 2$. For left cells $\omega, \omega^{\prime}$, we have

$$
\operatorname{Ext}_{\tilde{\mathcal{H}}}^{1}\left(\tilde{S}_{\omega}, \tilde{S}_{\omega^{\prime}}\right) \neq 0 \Rightarrow f(\omega)>f\left(\omega^{\prime}\right) .
$$

Proof. By Lemma 5.1 and Corollary 3.2 (which requires $e \neq 2$ ),

$$
\operatorname{Ext}_{\tilde{\mathcal{H}}}^{1}\left(\tilde{S}\left(\omega^{\prime}\right), \tilde{S}(\omega)\right) \neq 0 \Rightarrow f(\omega)>f\left(\omega^{\prime}\right) .
$$

For $\lambda \subseteq S$, the induced (right) $\mathcal{H}$-module $x_{\lambda} \mathcal{H}$ (see Section 1B) has an increasing filtration

$$
F_{\lambda}^{\cdot}: \quad 0=F_{\lambda}^{0} \subseteq F_{\lambda}^{1} \subseteq \cdots \subseteq F_{\lambda}^{m_{\lambda}}
$$


with sections $F_{\lambda}^{i+1} / F_{\lambda}^{i} \cong S_{\omega_{i}}$ and bottom section $F_{\lambda}^{1}=F_{\lambda}^{1} / F_{\lambda}^{0} \cong S_{\omega_{1}}$, where $\omega_{1}$ is the left cell containing the longest word $w_{\lambda, 0}$ in the parabolic subgroup $W_{\lambda}$. If $i>1$, then $\omega_{1}>_{L} \omega_{i}$; see [Du et al. 1998, (2.3.7)]. The indexing $\omega_{i}$ of (some of) the left cells depends on $\lambda$, and is formally "opposite" (in reverse order) to that used in [op. cit]. We write $\omega_{\lambda}:=\omega_{1}$ to denote its dependence of the latter cell on $\lambda$.

Lemma 5.4. In the filtration (5A.2), if $i>1$, then $f\left(\omega_{i}\right)>f\left(\omega_{\lambda}\right)$.

Proof. This follows from (2.7), since $\omega_{1}>_{L} \omega_{i}$ for all $2 \leq i \leq m_{\lambda}$, as noted above.

5B. First construction of a module $\tilde{X}_{\omega}$. Let $\omega \in \Omega$ be a fixed left cell. The construction of $\tilde{X}_{\omega}$ relies on Corollary 5.3.

We iteratively construct an $\tilde{\mathcal{H}}$-module $\tilde{X}_{\omega}$, filtered by dual left cell modules, such that $\tilde{S}_{\omega} \subseteq \tilde{X}_{\omega}$ is the lowest nonzero filtration term, and

$$
\operatorname{Ext}_{\tilde{\mathcal{H}}}^{1}\left(\tilde{S}_{\omega^{\prime}}, \tilde{X}_{\omega}\right)=0 \text { for all left cells } \omega^{\prime} .
$$

It will also be a consequence of the construction that every other filtration term $\tilde{S}_{\nu}$, $v \in \Omega$, satisfies $f(v)>f(\omega)$.

For $j \in \mathbb{N}$, let

$$
\Omega_{j}=\{v \in \Omega \mid f(v)=j\} .
$$

Fix $i=f(\omega)$. Suppose $\operatorname{Ext}_{\tilde{\mathcal{H}}}^{1}\left(\tilde{S}_{\tau}, \tilde{S}_{\omega}\right) \neq 0$ for some $\tau \in \Omega$. Then, by the Corollary 5.3, $f(\tau)>f(\omega)=i$. Assume $f(\tau)=j$ is minimal with this property. Since $\mathscr{Q}$ is a DVR and $\operatorname{Ext}_{\tilde{\mathcal{H}}}^{1}\left(\tilde{S}_{\tau}, \tilde{S}_{\omega}\right)$ is finitely generated, it follows that $\operatorname{Ext}_{\tilde{\mathcal{H}}}^{1}\left(\tilde{S}_{\tau}, \tilde{S}_{\omega}\right)$ is a direct sum of $m_{\tau}(\geq 0)$ nonzero cyclic $\mathscr{Q}$-modules. Let $\tilde{Y}_{\tau}$ be the extension of $\tilde{S}_{\tau}^{\oplus m_{\tau}}$ by $\tilde{S}_{\omega}$, constructed as above Lemma 4.1 (using generators for the cyclic modules). Then by Lemma 4.1, Corollary 4.2, and Corollary 5.3,

$$
\operatorname{Ext}_{\tilde{\mathcal{H}}}^{1}\left(\tilde{S}_{\tau}, \tilde{Y}_{\tau}\right)=0
$$

Let

$$
\Omega_{j, \omega}=\left\{v \in \Omega_{j} \mid \operatorname{Ext}_{\tilde{\mathcal{H}}}^{1}\left(\tilde{S}_{v}, \tilde{S}_{\omega}\right) \neq 0\right\} .
$$

If $v \in \Omega_{j, \omega} \backslash\{\tau\}$, then $\operatorname{Ext}_{\tilde{\mathcal{H}}}^{1}\left(\tilde{S}_{v}, \tilde{S}_{\omega}\right) \cong \operatorname{Ext}_{\tilde{\mathcal{H}}}^{1}\left(\tilde{S}_{v}, \tilde{Y}_{\tau}\right)$ by Corollary 5.3, together with the long exact sequence for Ext. ${ }^{5}$

Thus, if $\tilde{Y}_{\tau, v}$ denotes the corresponding extension of $\tilde{S}_{v}^{\oplus m_{\nu}}$ by $\tilde{Y}_{\tau}$ (again using the construction above Lemma 4.1), then

$$
\operatorname{Ext}_{\tilde{\mathcal{H}}}^{1}\left(\tilde{S}_{\omega^{\prime}}, \tilde{Y}_{\tau, v}\right)=0 \quad \text { for } \omega^{\prime}=\tau, v .
$$

${ }^{5}$ We also use the fact that $f(v) \neq f(\tau)$ implies that $\operatorname{Hom}_{\mathcal{H}}\left(\widetilde{S}_{\nu}, \widetilde{S}_{\tau}\right)=0$ since Hom ${ }_{\mathscr{Q}}\left(\tilde{S}_{v}, \widetilde{S}_{\tau}\right)$ and hence $\left.\operatorname{Hom}_{\mathcal{H}}\left(\widetilde{S}_{\nu}, \widetilde{S}_{\tau}\right)\right)$ are free $\mathcal{O}$-modules. Thus, if $\left.\operatorname{Hom}_{\mathcal{H}}\left(\widetilde{S}_{\nu}, \widetilde{S}_{\tau}\right)\right) \neq 0$, then it remains nonzero upon base change to $K$. This is impossible since $v$ and $\tau$ belong to different two-sided cells and $\tilde{\mathcal{H}}_{K}$ is semisimple. 
From the general identity

$$
\operatorname{Ext}_{\tilde{\mathcal{H}}}^{1}(A, C) \oplus \operatorname{Ext}_{\tilde{\mathcal{H}}}^{1}(B, C) \cong \operatorname{Ext}_{\tilde{\mathcal{H}}}^{1}(A \oplus B, C),
$$

one sees that $\tilde{Y}_{\tau, v}$ is isomorphic to the "sum" extension of $\tilde{S}_{\tau}^{\oplus m_{\tau}} \oplus \tilde{S}_{v}^{\oplus m_{v}}$ by $\tilde{S}_{\omega}$. Continuing this process, we obtain an extension $\tilde{Y}_{j}$ of $\bigoplus_{\tau \in \Omega_{j, \omega}} \tilde{S}_{\tau}^{\oplus m_{\tau}}$ by $\tilde{S}_{\omega}$, with

$$
\operatorname{Ext}_{\tilde{\mathcal{H}}}^{1}\left(\tilde{S}_{\omega^{\prime}}, \tilde{Y}_{j}\right)=0 \quad \text { for all } \omega^{\prime} \in \bigcup_{\ell \leq j} \Omega_{\ell} .
$$

Thus, $\operatorname{Ext}_{\tilde{\mathcal{H}}}^{1}\left(\tilde{S}_{\omega^{\prime}}, \tilde{Y}_{j}\right) \neq 0$ implies that $f\left(\omega^{\prime}\right)>j$.

Continuing the above construction with the role of $\tilde{S}_{\omega}$ replaced by $\tilde{Y}_{j_{1}}$ with $j_{1}=j$, we obtain a module $\tilde{Y}_{j_{1}, j_{2}}$ such that $j_{1}<j_{2}$ and

$$
\operatorname{Ext}_{\tilde{\mathcal{H}}}^{1}\left(\tilde{S}_{\omega^{\prime}}, \tilde{Y}_{j_{1}, j_{2}}\right)=0 \quad \text { for all } \omega^{\prime} \in \bigcup_{\ell \leq j_{2}} \Omega_{\ell} .
$$

Let $m$ be the maximal $f$-value. This construction will stop after a finite number $r=r(\omega)$ of steps, resulting in an $\tilde{\mathcal{H}}$-module $\tilde{X}_{\omega}:=\tilde{Y}_{j_{1}, j_{2}, \ldots, j_{r}}$ such that

$$
f(\omega)<j_{1}<j_{2}<\cdots<j_{r} \leq m, \quad \text { and } \operatorname{Ext}_{\tilde{\mathcal{H}}}^{1}\left(\tilde{S}_{\omega^{\prime}}, \tilde{X}_{\omega}\right)=0 \text { for all } \omega^{\prime} \in \Omega .
$$

5C. A second construction of a module $\tilde{X}_{\omega}$. The construction will generally lead to a larger module $\tilde{X}_{\omega}$, so is not as "efficient" as the first construction above, in some sense. Nevertheless, the construction has similar properties, is cleaner, and has the very considerable advantage that it first builds an $\mathcal{H}$-module $X_{\omega}$, then sets $\tilde{X}_{\omega}=X_{\omega, \mathscr{Q}}:=\left(X_{\omega}\right)_{\mathscr{Q}}$. Both $X_{\omega}$ and $\tilde{X}_{\omega}$ are built with the requirement $e \neq 2$, this condition being needed in the supporting Proposition 5.5(3) below.

As before, $\Omega$ denotes the set of all left cells of $W$, and $\Omega_{i}=\{\omega \in \Omega \mid f(\omega)=i\}$ for $i \in \mathbb{N}$.

Fix $\omega \in \Omega$, and put $i_{0}=f(\omega)$. For each $i \in \mathbb{Z}$, put $X_{\omega, i}=0$ if $i<i_{0}$ (we use these terms only as a notational convenience), and put $X_{\omega, i_{0}}=S_{\omega}$. Next, we give a recursive definition of $X_{\omega, j}$ for all $j \geq i_{0}$, with the case $j=i_{0}$ just given. If $X_{\omega, j}$ has been defined, define $X_{\omega, j+1}$ as follows: Let $M$ denote the direct sum (possibly zero) of all $\mathcal{H}$-modules $S_{\tau}$ with $f(\tau)=j+1$. Using the category $\mathcal{H}$-mod for $\mathscr{C}$ in the construction above Lemma 4.1, and $Y=X_{\omega, j}$, put $X_{\omega, j+1}=X$ in that construction (making some choice for the generators $\operatorname{Ext}_{\mathcal{H}}^{1}(M, Y)$ that are used). For $j$ sufficiently large, we have $\Omega_{i}=0$ for all $i>j$, and so $X_{\omega, i}=X_{\omega, j}$. Thus, we set $X_{\omega}:=X_{\omega, j}$ for any such sufficiently large $j$.

Proposition 5.5. The $\mathcal{H}$-module $X_{\omega}$ and the increasing filtration $\left\{X_{\omega, i}\right\}_{i \in \mathbb{Z}}$, constructed above, have the following properties:

(1) The smallest index of a nonzero section $X_{\omega, i} / X_{\omega, i-1}$ is $i=f(\omega)=i_{0}$, and the section is $S_{\omega}$ in that case. 
(2) All sections $X_{\omega, i} / X_{\omega, i-1}$ are direct sums of modules $S_{\tau}, \tau \in \Omega$, with varying multiplicities (possibly 0), and with $f(\tau)=i$.

(3) If $e \neq 2$, then $\operatorname{Ext}_{\tilde{\mathcal{H}}}^{1}\left(S_{\nu, \mathscr{Q}}, X_{\omega, \mathscr{Q}}\right)=0$ for all $v, \omega \in \Omega$.

Proof. Properties (1) and (2) are immediate from the construction of $X_{\omega}$.

To prove (3), fix $v$ and $\omega \in \Omega$. We will apply Corollary 5.3 several times. First, it shows the vanishing in (3) holds section by section of $X_{\omega, \mathscr{Q}}$, unless $f(v)>f(\omega)$. So assume that $f(v)>f(\omega)$.

Put $j=f(v)-1$ and let $M$ be the $\mathcal{H}$-module used above in the construction of $X_{\omega, j+1}$ from $Y=X_{\omega, j}$. Lemma 4.1 implies the map $\operatorname{Ext}_{\mathcal{H}}^{1}(M, Y) \rightarrow$ $\operatorname{Ext}_{\mathcal{H}}^{1}\left(M, X_{\omega, j+1}\right)$ is the zero map. Applying the flat base change from $\mathcal{Z}$ to $\mathscr{Q}$, we find that the map $\operatorname{Ext}_{\tilde{\mathcal{H}}}^{1}\left(M_{\mathscr{Q}}, Y_{\mathscr{Q}}\right) \rightarrow \operatorname{Ext}_{\tilde{\mathcal{H}}}^{1}\left(M_{\mathscr{Q}}, X_{\mathscr{Q}}\right)$ is zero, with $X=X_{\omega, j+1}$. However, Corollary 5.3 implies $\operatorname{Ext}_{\tilde{\mathcal{H}}}^{1}\left(M_{\mathscr{Q}}, M_{\mathscr{Q}}\right)=0$. Now the long exact sequence argument of Corollary 4.2 shows that $\operatorname{Ext}_{\tilde{\mathcal{H}}}^{1}\left(M_{\mathscr{Q}}, X_{\mathscr{Q}}\right)=0$. Since $S_{v}$ is a direct summand of $M$ (by construction, since $f(v)=j+1$ ), it follows that $\operatorname{Ext}_{\tilde{\mathcal{H}}}^{1}\left(S_{v, \mathscr{Q}}, X_{\mathscr{Q}}\right)=0$.

However, $X_{\omega} / X_{\omega, j+1}$ is filtered by modules $S_{\tau}$ with $f(\tau)>j+1=f(v)$. So

$$
\operatorname{Ext}_{\tilde{\mathcal{H}}}^{1}\left(S_{v, \mathscr{Q}},\left(X_{\omega} / X_{\omega, j+1}\right)_{\mathscr{Q}}\right)=0
$$

by Corollary 4.2 again. Together with the conclusion of the previous paragraph, this gives the required vanishing $\operatorname{Ext}_{\tilde{\mathcal{H}}}^{1}\left(S_{v, \mathscr{Q}}, X_{\omega, \mathscr{Q}}\right)=0$.

To complete the second construction, set $\tilde{X}_{\omega}=X_{\omega, \mathscr{Q}}$.

5D. The main result. Let $\Omega^{\prime}$ be the set of all left cells that do not contain the longest element of a parabolic subgroup. Put

$$
\tilde{\mathcal{T}}=\bigoplus_{\lambda \subseteq S} x_{\lambda} \tilde{\mathcal{H}} \quad \text { and } \quad \tilde{\mathcal{X}}=\bigoplus_{\omega \in \Omega^{\prime}} \tilde{X}_{\omega}
$$

Here and in the theorem below, objects (modules, algebras, etc.) are decorated with a tilde $\sim$ because they are taken over the DVR $\mathscr{Q}$ in (1C.1).

We are now ready to prove the following main result of the paper.

Theorem 5.6. Assume that $e \neq 2$. Let $\tilde{\mathcal{T}}^{+}=\tilde{\mathcal{T}} \oplus \tilde{\mathcal{X}}, \tilde{\mathcal{A}}^{+}=\operatorname{End}_{\tilde{\mathcal{H}}}\left(\tilde{\mathcal{T}}^{+}\right)$, and $\tilde{\Delta}(\omega)=\operatorname{Hom}_{\tilde{\mathcal{H}}}\left(\tilde{S}_{\omega}, \tilde{\mathcal{T}}^{+}\right)$for $\omega \in \Omega$. Then $\{\tilde{\Delta}(\omega)\}_{\omega \in \Omega}$ is a strict stratifying system for the category $\tilde{\mathcal{A}}^{+}$-mod with respect to the quasi-poset $\left(\Omega, \leq_{f}\right)$.

Proof. For each left cell $\omega$, put $\tilde{T}_{\omega}=x_{\lambda} \tilde{\mathcal{H}}$ if $\omega$ contains the longest element $w_{\lambda, 0}$ of $W_{\lambda}$, where $\lambda \subseteq S$. If there is no such $\lambda$ for $\omega$, put $\tilde{T}_{\omega}=\tilde{X}_{\omega}$ as constructed in Section 5B. (One can use the $\tilde{X}_{\omega}$ from Section $5 \mathrm{C}$ with slight adjustments, left to the reader.) In the first case, $\tilde{T}_{\omega}$ has a filtration by dual left cell modules, and $\tilde{S}_{\omega}$ appears at the bottom. Moreover, $f(\omega)<f\left(\omega^{\prime}\right)$ for any other filtration section $\tilde{S}_{\omega^{\prime}}$, by Lemma 5.4 . This same property holds also in the case $\tilde{T}_{\omega}=\tilde{X}_{\omega}$ by construction. 
Put $\tilde{T}=\bigoplus_{\omega} \tilde{T}_{\omega}$ and note $\tilde{\mathcal{T}}^{+}=\tilde{T}$. We will apply Theorem 1.1 to $\tilde{T}$ and the various $\tilde{T}_{\omega}$, where $\tilde{\mathcal{H}}$ plays the role of the algebra $B$ there, $\mathscr{Q}$ plays the role of $R$ there, $\tilde{S}_{\omega}$ is $S_{\lambda}$, etc. We are required to the check three conditions (1), (2), (3) in Theorem 1.1. The construction in Section 5B of dual left cell filtrations of the various $\tilde{T}_{\omega}$ is precisely what is required for the verification of (1).

Condition (2) translates directly to the requirement

$$
\operatorname{Hom}_{\tilde{\mathcal{H}}}\left(\tilde{S}_{\mu}, \tilde{T}_{\omega}\right) \neq 0 \Rightarrow \omega \leq_{f} \mu
$$

for given $\mu, \omega$. However, if $\operatorname{Hom}_{\tilde{\mathcal{H}}}\left(\tilde{S}_{\mu}, \tilde{T}_{\omega}\right) \neq 0$, then there must be a nonzero $\operatorname{Hom}_{\tilde{\mathcal{H}}}\left(\tilde{S}_{\mu}, \tilde{S}_{\omega^{\prime}}\right)$ for some filtration section $\tilde{S}_{\omega^{\prime}}$ of $\tilde{T}_{\omega}$. In particular, $f\left(\omega^{\prime}\right) \geq f(\omega)$. Also, $\left(\tilde{S}_{\mu}\right)_{K}$ and $\left(\tilde{S}_{\omega^{\prime}}\right)_{K}$ must have a common irreducible constituent, forcing the two-sided cells containing $\mu$ and $\omega^{\prime}$ to agree. This gives $f(\mu)=f\left(\omega^{\prime}\right) \geq f(\omega)$; so (2) holds.

Finally,

$$
\operatorname{Ext}_{\tilde{\mathcal{H}}}^{1}\left(\tilde{S}_{\mu}, \tilde{T}_{\omega}\right)=0 \quad \text { for all } \mu, \omega \text {. }
$$

This follows from the construction Section $5 \mathrm{~B}$ for $\tilde{T}_{\omega}=\tilde{X}_{\omega}$ and by Corollary 4.5 in case $\tilde{T}_{\omega}=x_{\lambda} \tilde{\mathcal{H}}$. The conclusion of Theorem 1.1 now immediately gives the theorem we are proving here.

\section{Identification of $\tilde{\mathcal{A}}^{+}=\operatorname{End}_{\tilde{\mathcal{H}}}\left(\tilde{\mathcal{T}}^{+}\right)$}

The constructions in Section $5 \mathrm{~B}$ of the modules $\tilde{X}_{\omega}$ in the previous section work just as well using the modules $\tilde{S}_{E}:=\tilde{S}(E)^{*}$ for $E \in \operatorname{Irr}(\mathbb{Q} W)$ defined in (3A.5) to replace the dual left cell modules $\tilde{S}_{\omega}$. This results in right $\mathcal{H}$-modules $\tilde{X}_{E}$. As in the case of $\tilde{X}_{\omega}$, we have the following property, with the same proof. In the statement of the following proposition, $\tilde{X}_{E}$ can be defined using either of the two constructions.

Proposition 6.1. If $e \neq 2$, then $\operatorname{Ext}_{\tilde{\mathcal{H}}}^{1}\left(\tilde{S}_{E^{\prime}}, \tilde{X}_{E}\right)=0$ for all $E, E^{\prime} \in \operatorname{Irr}(\mathbb{Q} W)$.

If we use the first construction given in Section $5 \mathrm{~B}$, the modules $\tilde{X}_{E}$ have strong indecomposability properties, which the modules $\tilde{X}_{\omega}, \omega \in \Omega$, generally do not have with either construction. In the following proposition, we assume that $\tilde{X}_{E}$ is defined by the first construction Section 5B.

The following result can be argued without using RDAHAs, but it is faster to quote Rouquier's 1-faithful covering theory, especially [Rouquier 2008, Theorem 5.3], which applies to our $e \neq 2$ case, over $\mathscr{R}$, where

$$
\mathscr{R}:=\left(\mathbb{C}\left[t, t^{-1}\right]_{(t-\sqrt{\zeta})}\right)^{\wedge}
$$


is the completion of the localization $\mathbb{C}\left[t, t^{-1}\right]_{(t-\sqrt{\zeta})}$ at the maximal ideal $(t-\sqrt{\zeta})$. Note that $\mathscr{R}$ is a $\mathscr{Q}$-module via the natural ring homomorphism $\mathscr{Q} \rightarrow \mathscr{R}$. Note also that the set $\operatorname{Irr}(\mathbb{Q} W)$ corresponds naturally to the set $\operatorname{Irr}(W):=\operatorname{Irr}(\mathbb{C} W)$ in [op. cit].

Proposition 6.2. Assume that $e \neq 2$. The right $\tilde{\mathcal{H}}$-modules $\tilde{X}_{E}$ are indecomposable, as is each $\tilde{X}_{E} \otimes k$. The endomorphism algebras of all these modules are local with radical quotient $k$.

Proof. It is clear that $\tilde{X}_{E, \mathscr{R}}=\tilde{X}_{E} \otimes_{\mathscr{Q}} \mathscr{R}$ can be constructed from $\tilde{S}_{E, \mathscr{R}}$ in the same way that $\tilde{X}_{E}$ is constructed from $\tilde{S}_{E}$, again using the method of Section 5B. Also, the proof of [op. cit, Theorem 6.8] shows that the $\mathscr{R}$-dual of $\tilde{S}_{E, \mathscr{R}}$ is the KZ-image of the standard module $\Delta_{\mathscr{R}}(E)$ in the $\mathscr{R}$-version of $\mathcal{O}$. (Recall the issues in footnote 2.)

Consequently, by the 1 -faithful property, $\left(\tilde{X}_{E, \mathscr{R}}\right)^{*}$ is the image of a dually constructed module $P$ under the functor KZ, filtered by standard modules, and with $\operatorname{Ext}_{\mathcal{O}}^{1}(P,-)$ vanishing on all standard modules. Such a module $P$ is projective in $\mathcal{O}$, by [op. cit, Lemma 4.22]. (We remark that both $\mathcal{O}$ and $\mathrm{KZ}$ would be given a subscript $\mathscr{R}$ in [Ginzburg et al. 2003] though not in [Rouquier 2008].)

If we knew $P$ were indecomposable, we could say $\tilde{X}_{E, \mathscr{R}}$ is indecomposable. However, the indecomposability of $P$ requires proof. ${ }^{6}$ We do this by showing $P$ is the projective cover in $\mathcal{O}$ of the standard module $\Delta(E)=\Delta_{\mathcal{O}}(E)$. We can, instead, inductively show the truncation $P_{i}$, associated to the poset ideal of all $E^{\prime} \in \operatorname{Irr}(\mathbb{Q} W)$ with $f\left(E^{\prime}\right) \leq i$, is the projective cover of $\Delta(E)$ in the associated truncation $\mathcal{O}_{i}$ of $\mathcal{O}$. This requires $\Delta(E)$ to be an object of $\mathcal{O}_{i}$, or equivalently $f(E) \leq i$.

If $f(E)=i$, then $P_{i}=\Delta(E)$ is trivially the projective cover of $\Delta(E)$. Inductively, $P_{i-1}$ is the projective cover of $\Delta(E)$ in $\mathcal{O}_{i-1}$ for some $i>f(E)$. Let $P^{\prime}$ denote the projective cover of $\Delta(E)$ in $\mathcal{O}_{i}$. The truncation $\left(P^{\prime}\right)_{i-1}$ to $\mathcal{O}_{i-1}$ of $P^{\prime}$ - that is, its largest quotient which is an object of $\mathcal{O}_{i-1}-$ is clearly isomorphic to $P_{i-1}$. Let $\theta: P^{\prime} \rightarrow P_{i}$ be a homomorphism extending a given isomorphism $\psi:\left(P^{\prime}\right)_{i-1} \rightarrow P_{i-1}$ and let $\tau: P_{i} \rightarrow P^{\prime}$ be a homomorphism extending $\psi^{-1}$. Let $M, M^{\prime}$ denote the kernels of the natural surjections $P_{i} \rightarrow P_{i-1}$ and $P^{\prime} \rightarrow\left(P^{\prime}\right)_{i-1}$. The map $\tau \theta: P^{\prime} \rightarrow P^{\prime}$ is surjective and, consequently, it is an isomorphism. It induces the identity on $\left(P^{\prime}\right)_{i-1}$. Therefore, the induced map

$$
\left.\left.\tau\right|_{M} \theta\right|_{M^{\prime}}: M^{\prime} \longrightarrow M^{\prime}
$$

is an isomorphism, and $M=M^{\prime} \oplus M^{\prime \prime}$ for some object $M^{\prime \prime}$ in $\mathcal{O}$. By construction, $M$ is a direct sum of objects $\Delta\left(E^{\prime}\right)$, with $f\left(E^{\prime}\right)=i$, each appearing with multiplicity $m_{E^{\prime}}=\operatorname{rank}_{\operatorname{Ext}_{\mathcal{O}}^{1}}^{1}\left(P_{i}, \Delta\left(E^{\prime}\right)\right)$. However,

$$
\operatorname{Ext}_{\mathcal{O}}^{1}\left(P_{i-1}, \Delta\left(E^{\prime}\right)\right) \cong \operatorname{Hom}_{\mathcal{O}}\left(M^{\prime}, \Delta\left(E^{\prime}\right)\right) .
$$

${ }^{6} \mathrm{~A}$ similar point should be made regarding the uniqueness claim in [Rouquier 2008, Proposition 4.45], which is false without a minimality assumption on $Y(M)$ there. 
It follows that $M^{\prime \prime}=0$ and $P_{i} \cong P^{\prime}$ is indecomposable.

In particular, $P$ is indecomposable and consequently $\tilde{X}_{E, \mathscr{R}}$ is indecomposable, as noted. In turn, this implies $\tilde{X}_{E}$ is indecomposable. The 0 -faithfulness (or just the covering property itself) of the cover given by $\mathcal{O}$ and $\mathrm{KZ}$ imply

$$
\operatorname{End}_{\tilde{\mathcal{H}}_{\mathscr{R}}}\left(\tilde{X}_{E, \mathscr{R}}\right)^{\mathrm{op}} \cong \operatorname{End}_{\tilde{\mathcal{H}}_{\mathscr{R}}}\left(\tilde{X}_{E, \mathscr{R}}^{*}\right)^{\mathrm{op}} \cong \operatorname{End}_{\mathcal{O}}(P) .
$$

Thus, the base-changed module $P \otimes_{\mathscr{R}} \mathbb{C}$ has endomorphism ring

$$
\operatorname{End}_{\mathcal{O}_{\mathbb{C}}}\left(P \otimes_{\mathscr{R}} \mathbb{C}\right) \cong \operatorname{End}_{\mathcal{O}}(P) \otimes_{\mathscr{R}} \mathbb{C},
$$

where $\mathcal{O}_{\mathbb{C}}$ is the $\mathbb{C}$-version of $\mathcal{O}$. This is a standard consequence of the projectivity of $P$. By [Rouquier 2008, Theorem 5.3], the $\mathbb{C}$ versions of $\mathrm{KZ}$ and $\mathcal{O}$ give a cover for $\tilde{\mathcal{H}}_{\mathscr{R}} \otimes \mathbb{C}$. So $\operatorname{End}_{\tilde{\mathcal{H}}_{\mathbb{C}}}\left(\tilde{X}_{E, \mathscr{R}} \otimes \mathbb{C}\right)^{\text {op }} \cong \operatorname{End}_{\mathcal{O}_{\mathbb{C}}}(P \otimes \mathbb{C})$ is local, with radical quotient $\mathbb{C}$.

However, we have

$$
\left(\tilde{X}_{E} \otimes_{\mathscr{Q}} k\right) \otimes_{k} \mathbb{C} \cong \tilde{X}_{E, \mathscr{R}} \otimes \mathbb{C} .
$$

In particular, $\tilde{X}_{E} \otimes_{\mathscr{Q}} k$ is indecomposable since (by endomorphism ring considerations) the $\tilde{\mathcal{H}}_{\mathscr{R}} \otimes \mathbb{C}$-module $\tilde{X}_{E, \mathscr{R}} \otimes \mathbb{C}$ is indecomposable. So the endomorphism ring of $\tilde{X}_{E} \otimes_{\mathscr{Q}} k$ over the finite dimensional algebra $\tilde{\mathcal{H}} \otimes_{\mathscr{Q}} k$ is local. The radical quotient is a division algebra $D$ over $k$ with base change $-\otimes_{k} \mathbb{C}$ to a semisimple quotient of $\operatorname{End}_{\tilde{\mathcal{H}}_{\mathbb{C}}}\left(\tilde{X}_{E, \mathscr{R}} \otimes \mathbb{C}\right)$, which could only be $\mathbb{C}$ itself. Consequently, $D=k$.

Finally, the vanishing $\operatorname{Ext}_{\tilde{\mathcal{H}}}^{1}\left(\tilde{X}_{E}, \tilde{X}_{E}\right)=0$ implies

$$
\operatorname{End}_{\tilde{\mathcal{H}}}\left(\tilde{X}_{E}\right) \otimes_{\mathscr{Q}} k \cong \operatorname{End}_{\tilde{\mathcal{H}}_{k}}\left(\tilde{X}_{E} \otimes_{\mathscr{Q}} k\right) .
$$

So the $\operatorname{ring} \operatorname{End}_{\tilde{\mathcal{H}}}\left(\tilde{X}_{E}\right)$ is local with radical quotient $k$. This completes the proof.

Lemma 6.3. Assume $e \neq 2$. Let $E \in \operatorname{Irr}(\mathbb{Q} W)$. Then $\tilde{X}_{E}$ is a direct summand of $\tilde{\mathcal{T}}^{+}$. Proof. Suppose first that $\tilde{S}(E)$ is a direct summand of a left cell module $\tilde{S}(\omega) \cong$ $\left(\tilde{S}_{\omega}\right)^{*}$, where $\omega$ contains the longest element of a parabolic subgroup $W_{\lambda}$, for $\lambda \subseteq S$. This implies $\tilde{S}_{\omega}$ is the lowest term in the dual left cell module filtration of $x_{\lambda} \tilde{\mathcal{H}}$. Consequently, there is an inclusion $\psi: \tilde{S}_{E} \hookrightarrow x_{\lambda} \tilde{\mathcal{H}}$ with cokernel filtered by (sections) $\tilde{S}_{E^{\prime}}, E^{\prime} \in \operatorname{Irr}(\mathbb{Q} W)$. Thus, $\psi^{-1}: \psi\left(\tilde{S}_{E}\right) \rightarrow \tilde{X}_{E}$ may be extended to a map $\phi: x_{\lambda} \tilde{\mathcal{H}} \rightarrow \tilde{X}_{E}$ of $\tilde{\mathcal{H}}$-modules. Similarly (using $e \neq 2$ and Corollary 4.5), there is a map $\tau: \tilde{X}_{E} \rightarrow x_{\lambda} \tilde{\mathcal{H}}$ extending $\psi$. The composite $\tau \phi$ restricts to the identity on $\tilde{S}_{E} \subseteq \tilde{X}_{E}$

On the other hand, restriction from $\tilde{X}_{E}$ to $\tilde{S}_{E}$ defines a homomorphism

$$
\operatorname{End}_{\tilde{\mathcal{H}}}\left(\tilde{X}_{E}\right) \longrightarrow \operatorname{End}_{\tilde{\mathcal{H}}}\left(\tilde{S}_{E}\right)
$$

since $\left(\tilde{S}_{E}\right)_{K}$ is a unique summand of the (completely reducible) $\tilde{\mathcal{H}} \otimes_{\mathscr{Q}} K$-module $\tilde{X}_{E} \otimes_{\mathscr{Q}} K$. (Observe $\tilde{S}_{E}=\tilde{X}_{E} \cap\left(\tilde{S}_{E}\right)_{K}$, since the $\mathscr{Q}$-torsion module $\left(\tilde{X}_{E} \cap\left(\tilde{S}_{E}\right)_{K}\right) / \tilde{S}_{E}$ 
must be zero in the $\mathscr{Q}$-torsion free module $\tilde{X}_{E} / \tilde{S}_{E}$.) Thus, $\tau \phi$ is a unit in the local endomorphism $\operatorname{ring} \operatorname{End}_{\tilde{\mathcal{H}}}\left(\tilde{X}_{E}\right)$, so $\tilde{X}_{E}$ is a summand of $x_{\lambda} \tilde{\mathcal{H}}$, and hence of $\tilde{\mathcal{T}}$.

Next consider the case in which $\tilde{S}_{E}$ is a summand of a dual left cell module $\tilde{S}_{\omega}$ (this always happens for some $\omega$ ), but $\omega$ does not contain the longest element of any parabolic subgroup. In this case, $\tilde{X}_{\omega}$ is one of the summands of $\tilde{\mathcal{X}}$ by construction. The argument above may be repeated with $\tilde{X}_{\omega}$ playing the role of $x_{\lambda} \tilde{\mathcal{H}}$. In the same way, $\tilde{X}_{E}$ is a direct summand of $\tilde{X}_{\omega}$, and thus of $\tilde{\mathcal{X}}$.

In both cases, we conclude that $\tilde{X}_{E}$ is a direct summand of $\tilde{\mathcal{T}} \oplus \tilde{\mathcal{X}}=\tilde{\mathcal{T}}^{+}$.

Theorem 6.4. Assume that $e \neq 2$. The $\mathscr{Q}$-algebra $\tilde{\mathcal{A}}^{+}$is quasi-hereditary, with standard modules $\tilde{\Delta}(E)=\operatorname{Hom}_{\tilde{\mathcal{H}}}\left(\tilde{S}_{E}, \tilde{\mathcal{T}}^{+}\right), E \in \operatorname{Irr}(\mathbb{Q} W)$, and partial order $<_{f}$.

Proof. We have already seen that this algebra is standardly stratified with strict stratifying system $\{\tilde{\Delta}(\omega)\}_{\omega \in \Omega}$. Clearly, $\tilde{\Delta}(\omega)$ is a direct sum of various $\tilde{\Delta}(E)$, and every $\tilde{\Delta}(E)$ arises as such a summand.

Put $\tilde{P}(E)=\left(\tilde{X}_{E}\right)^{\diamond}:=\operatorname{Hom}_{\tilde{\mathcal{H}}^{(}}\left(\tilde{X}_{E}, \tilde{\mathcal{T}}^{+}\right), E \in \operatorname{Irr}(\mathbb{Q} W)$. Then $\tilde{P}(E)$ is a direct summand of $\tilde{\mathcal{A}}^{+}=\operatorname{End}_{\tilde{\mathcal{H}}}\left(\tilde{\mathcal{T}}^{+}\right)$, viewed as a left module over itself. Thus, $\tilde{P}(E)$ is projective as an $\tilde{\mathcal{A}}^{+}$-module, and $\tilde{P}(E)^{\diamond}:=\operatorname{Hom}_{\tilde{\mathcal{A}}^{+}}\left(\tilde{P}(E), \tilde{\mathcal{T}}^{+}\right)$is naturally isomorphic to $\tilde{X}_{E}$. In particular, the contravariant functor $(-)^{\diamond}$ gives an isomorphism

$$
\operatorname{End}_{\tilde{\mathcal{A}}^{+}}(\tilde{P}(E)) \cong\left(\operatorname{End}_{\tilde{\mathcal{H}}}\left(\tilde{X}_{E}\right)\right)^{\mathrm{op}} .
$$

Consequently, $\tilde{P}(E)$ also has a local endomorphism ring with radical quotient $k$, as does $\operatorname{End}_{\tilde{\mathcal{A}}_{k}^{+}}\left(\tilde{P}(E) \otimes_{\mathscr{Q}} k\right)$. It follows that $\tilde{P}(E)$ is an indecomposable projective $\tilde{\mathcal{A}}^{+}$-module with a irreducible head. (The arguments in this paragraph are largely standard, many taken from [Du et al. 1998].)

By (5D.1), $\operatorname{Ext}_{\tilde{\mathcal{H}}^{1}}^{1}\left(\tilde{S}_{\omega}, \tilde{\mathcal{T}}^{+}\right)=0$ for all dual left cell module $\tilde{S}_{\omega}$. Consequently, a similar vanishing holds with $\tilde{S}_{\omega}$ replaced by any module $\tilde{S}_{E^{\prime}}, E^{\prime} \in \operatorname{Irr}(\mathbb{Q} W)$. It follows that the restriction map

$$
\tilde{P}(E)=\operatorname{Hom}_{\tilde{\mathcal{H}}}\left(\tilde{X}_{E}, \tilde{\mathcal{T}}^{+}\right) \longrightarrow \operatorname{Hom}_{\tilde{\mathcal{H}}}\left(\tilde{S}_{E}, \tilde{\mathcal{T}}^{+}\right)=\tilde{\Delta}(E)
$$

is surjective. Hence, $\tilde{\Delta}(E)$ has an irreducible head. Also, repeating the argument for filtered submodules of $\tilde{X}_{E}$, we find that the kernel of the above map has a filtration with sections $\tilde{\Delta}\left(E^{\prime}\right), E^{\prime} \in \operatorname{Irr}(\mathbb{Q} W)$ (rather than $\tilde{X}_{E}$ itself), satisfying $f\left(E^{\prime}\right)>f(E)$.

Next, we claim that $\tilde{\Delta}(E)^{\diamond}:=\operatorname{Hom}_{\tilde{\mathcal{A}}^{+}}\left(\tilde{\Delta}(E), \tilde{\mathcal{T}}^{+}\right)$is naturally isomorphic to $\tilde{S}_{E}$. More precisely, we claim that the natural map ev : $\tilde{S}_{E} \rightarrow\left(\tilde{S}_{E}\right)^{\diamond \diamond}$ is an isomorphism. We showed above that the sequence

$$
0 \longrightarrow\left(\tilde{X}_{E} / \tilde{S}_{E}\right)^{\diamond} \longrightarrow\left(\tilde{X}_{E}\right)^{\diamond} \longrightarrow\left(\tilde{S}_{E}\right)^{\diamond} \longrightarrow 0
$$

is exact. Applying $(-)^{\diamond}$ once more, we get an injection

$$
0 \longrightarrow\left(\tilde{S}_{E}\right)^{\diamond \diamond} \longrightarrow\left(\tilde{X}_{E}\right)^{\diamond \diamond}
$$


with $\tilde{X}_{E} \stackrel{\text { ev }}{\rightarrow}\left(\tilde{X}_{E}\right)^{\diamond \diamond}$ an isomorphism. This gives inclusions

$$
\tilde{S}_{E} \cong \operatorname{ev}\left(\tilde{S}_{E}\right) \subseteq\left(\tilde{S}_{E}\right)^{\diamond \diamond} \subseteq\left(\tilde{X}_{E}\right)^{\diamond \diamond} \cong \tilde{X}_{E} .
$$

If $(-) \otimes_{\mathscr{Q}} K$ is applied, the first inclusion becomes an isomorphism. This gives

$$
\left(\tilde{S}_{E}\right)^{\diamond \diamond} \subseteq\left(\tilde{X}_{E}\right)^{\diamond \diamond} \cap\left(\tilde{S}_{E}\right)_{K}=\tilde{S}_{E}
$$

identifying $\tilde{X}_{E}$ with $\left(\tilde{X}_{E}\right)^{\diamond \diamond}$ and $\tilde{S}_{E}$ with its image in $\left(\tilde{X}_{E}\right)^{\diamond \diamond}$. Consequently, $\operatorname{ev}\left(\tilde{S}_{E}\right)=\left(\tilde{S}_{E}\right)^{\diamond \diamond}$, proving the claim.

Finally, we suppose $E ¥ E^{\prime} \in \operatorname{Irr}(\mathbb{Q} W)$ and $\operatorname{Hom}_{\tilde{\mathcal{A}}^{+}}\left(\tilde{P}\left(E^{\prime}\right), \tilde{\Delta}(E)\right) \neq 0$. Using the identifications $\tilde{P}\left(E^{\prime}\right)=\left(\tilde{X}_{E^{\prime}}\right)^{\diamond}, \tilde{\Delta}(E)=\left(\tilde{S}_{E}\right)^{\diamond}, \tilde{P}\left(E^{\prime}\right)^{\diamond} \cong \tilde{X}_{E^{\prime}}$, and $\tilde{\Delta}(E)^{\diamond} \cong \tilde{S}_{E}$, we have

$$
0 \neq \operatorname{Hom}_{\tilde{\mathcal{A}}^{+}}\left(\tilde{P}\left(E^{\prime}\right), \tilde{\Delta}(E)\right) \cong \operatorname{Hom}_{\tilde{\mathcal{H}}}\left(\tilde{S}_{E}, \tilde{X}_{E^{\prime}}\right) \subseteq \operatorname{Hom}_{\tilde{\mathcal{H}}_{K}}\left(\tilde{S}_{E} \otimes_{\mathscr{Q}} K, \tilde{X}_{E^{\prime}} \otimes_{\mathscr{Q}} K\right) .
$$

This implies $f\left(E^{\prime}\right)<f(E)$. It follows now from [Du et al. 1998, Theorem 1.2.8] (in the context of stratified algebras), [Du and Scott 1994, Corollary 2.5], or [Rouquier 2008, Theorem 4.16] that $\tilde{\mathcal{A}}^{+}$is quasi-hereditary over $\mathscr{Q}$.

We are now ready to establish the category equivalence mentioned in the introduction. Again, we use the covering theory of [Rouquier 2008].

Theorem 6.5. Assume that $e \neq 2$. The category of left modules over the basechanged algebra

$$
\tilde{\mathcal{A}}_{\mathscr{R}}^{+}:=\tilde{\mathcal{A}}^{+} \otimes_{\mathscr{Q}} \mathscr{R}
$$

is equivalent to the $\mathscr{R}$-category $\mathcal{O}$ of modules, as defined in [Rouquier 2008] for the RDAHA associated to $W$ over $\mathscr{R}$.

Proof. Continuing the proof of the theorem above, the projective indecomposable $\tilde{\mathcal{A}}^{+}$-modules are the various $\tilde{P}(E)=\left(\tilde{X}_{E}\right)^{\diamond}$. Consequently, $\tilde{\mathcal{T}}^{+}=\left(\tilde{\mathcal{A}}^{+}\right)^{\diamond}$ is the direct sum of the modules, $\tilde{X}_{E}$, each with nonzero multiplicities. The modules $\tilde{X}_{E, \mathscr{R}}$ remain indecomposable, as observed in the proof of the indecomposability of the modules $\tilde{X}_{E}$ above. By construction, $\operatorname{Ext}_{\tilde{\mathcal{H}}}^{1}\left(\tilde{S}_{E^{\prime}}, \tilde{X}_{E}\right)=0$ for all $E, E^{\prime} \in \operatorname{Irr}(\mathbb{Q} W)$. Thus, there is a similar vanishing for $\tilde{S}_{E^{\prime}, \mathscr{R}}$ and $\tilde{X}_{E, \mathscr{R}}$, and - in the reverse orderfor their $\mathscr{R}$-linear duals. Observe that $\left(\tilde{S}_{E^{\prime}, \mathscr{R}}\right)^{*} \cong \tilde{S}\left(E^{\prime}\right) \otimes_{\mathscr{Q}} \mathscr{R}$ is $\mathrm{KZ}\left(\Delta\left(E^{\prime}\right)\right)$, taking $\Delta\left(E^{\prime}\right)=\Delta_{\mathcal{O}}\left(E^{\prime}\right)$ to be the standard module for the category $\mathcal{O}$ over $\mathscr{R}$ as discussed in [loc. cit.] together with $\mathrm{KZ}$ for this category.

Put

$$
Y=\bigoplus_{E}\left(\tilde{X}_{E, \mathscr{R}}\right)^{*}
$$

and set $Y\left(\tilde{S}_{E, \mathscr{R}}^{*}\right)=\left(\tilde{X}_{E, \mathscr{R}}\right)^{*}$. This notation imitates that of [op. cit, Proposition 4.45]. The first part of this proposition is missing a necessary minimality assumption 
on the rank of $Y(M)$, in the terminology there. ${ }^{7}$ However, this is satisfied for $M=\left(\tilde{S}_{E, \mathscr{R}}\right)^{*}$ and $Y(M)=\left(\tilde{X}_{E, \mathscr{R}}\right)^{*}$ because $\left(\tilde{X}_{E, \mathscr{R}}\right)^{*}$ is indecomposable. Several other corrections, in addition to the minimality requirement, should be made to [op. cit, Proposition 4.45]:

- $A^{\prime}$ should be redefined as $\operatorname{End}_{B}(Y)^{\mathrm{op}}$;

- $P^{\prime}$ should be redefined as $\operatorname{Hom}_{B}(Y, B)^{\mathrm{op}}$.

In addition, $B$ in [op. cit, §4.2.1] should be redefined as $\operatorname{End}_{A}(P)^{\text {op }}$. The instances of "op" here and above insure action on the left, and consistency with [Ginzburg et al. 2003, Theorems 5.14 and 5.15]. The definition of $P^{\prime}$ is given to be consistent with the basis covering property $\operatorname{End}_{A^{\prime}}\left(P^{\prime}\right)^{\mathrm{op}} \cong B$, as in [loc. cit.] — we do not need this fact below.

With these changes, [Rouquier 2008, Theorem 5.3, Proposition 4.45, and Corollary 4.46] guarantees that $\mathcal{A}^{\prime}$-mod is equivalent to $\mathcal{O}$, where $\mathcal{A}^{\prime}=\operatorname{End}_{\tilde{\mathcal{H}} \mathscr{R}}(Y)$. (All we really need for this are the 0 - and 1-faithfulness of the $\mathcal{O}$ version of the $\mathrm{KZ}$ functor.) However, $\operatorname{End}_{\tilde{\mathcal{H}}_{\mathscr{R}}}(Y) \cong \operatorname{End}_{\tilde{\mathcal{H}}_{\mathscr{R}}}\left(Y^{*}\right)^{\text {op }}$, and $Y^{*}$ is the direct sum $\bigoplus_{E} \tilde{X}_{E, \mathscr{R}}$. Hence,

$$
Y^{* \diamond} \cong \bigoplus_{E}\left(\tilde{X}_{E, \mathscr{R}}\right)^{\diamond} \cong \bigoplus_{E} \tilde{P}(E) \otimes_{\mathscr{Q}} \mathscr{R}
$$

Recall that $\left(\tilde{X}_{E, \mathscr{R}}\right)^{\diamond \diamond} \cong \tilde{X}_{E, \mathscr{R}}$, so that the analogous property holds for $Y^{*}$. Thus,

$$
\operatorname{End}_{\tilde{\mathcal{H}}_{\mathscr{R}}}\left(Y^{*}\right)^{\mathrm{op}} \cong \operatorname{End}_{\tilde{\mathcal{A}}_{\mathscr{R}}^{+}}\left(Y^{* \diamond}\right) .
$$

Since the module $Y^{* \diamond}$ as displayed above is clearly a projective generator for $\tilde{\mathcal{A}}_{\mathscr{R}}^{+}$, there is a Morita equivalence over $\mathscr{R}$ of $\tilde{\mathcal{A}}_{\mathscr{R}}^{+}$with $\mathcal{A}^{\prime}$. Hence, $\tilde{\mathcal{A}}_{\mathscr{R}}^{+}$-mod is equivalent to $\mathcal{O}$, as $\mathscr{R}$-categories.

\section{Appendix: comparison with [Du et al. 1998, Conjecture 2.5.2]}

Conjecture 1.2 in this paper retains the most essential features of [Du et al. 1998, Conjecture 2.5.2], but is more flexible. In particular:

(1) Conjecture 1.2 does not specify the preorder $\leq$, only requiring that it be strictly compatible with the partition of $\Omega$ into two-sided cells. This allows the use of the preorder $\leq_{f}$, defined in Section 2 above. [Du et al. 1998] specifies for $\leq$ the preorder $\leq_{L R}^{\mathrm{op}}$ built from the preorder $\leq_{L R}$ originally used by Kazhdan-Lusztig to define the two-sided cells. In both cases, the set $\bar{\Omega}$ of "strata" is the same, identifying with the set of two sided cells.

${ }^{7}$ The proposition claims uniqueness for a pair $\left(Y(M), p_{m}\right)$. However, one gets another pair by adding a direct summand $F(P)$ to the kernel of $p_{m}$, where $P$ is any finitely generated module in the highest weight category $\mathscr{C}$. 
(2) Conjecture 1.2 concerns the Hecke algebra $\mathcal{H}$ (defined by the relations (1.1) over $\mathcal{Z}=\mathbb{Z}\left[t, t^{-1}\right]$, whereas [Du et al. 1998, Conj. 2.5.2] uses Hecke algebras over $\mathbb{Z}\left[t^{2}, t^{-2}\right]$. Largely, this change has been made to conform to the literature, which most often uses the former ring. There is an additional advantage that the quotient field $\mathbb{Q}(t)$ is almost always a splitting field for the Hecke algebra $\mathcal{H}_{\mathbb{Q}(t)}$. Note that $\mathbb{Q}(t)$ is always a splitting field in case the rank is greater than 2 . In the rank 2 case of ${ }^{2} F_{4}, \mathcal{H}_{\mathbb{Q}(t)}$ splits after $\sqrt{2}$ is adjoined. The conjecture in all rank 2 cases follows from [Du et al. 1998, §3.5].

(3) The role of $\mathcal{A}_{R}^{+}$in Conjecture 1.2 is played by $\operatorname{End}_{\mathcal{H}_{R}}\left(\mathcal{T}_{R}^{+}\right)$in [Du et al. 1998, Conjecture 2.5.2]. The two $R$-algebras are the same whenever $R$ is flat over $\mathcal{Z}=\mathbb{Z}\left[t, t^{-1}\right]$. While it is an interesting question as to whether or not such a base change property holds for any $\mathcal{Z}$-algebra $R$, it seems best to separate this issue from the main stratification proposal of the conjecture.

Finally, we mention that the original conjecture [Du et al. 1998, Conjecture 2.5.2] was checked in that paper for all rank two types (in both the equal and unequal parameter cases), and checked later in type $A$ for all ranks; see [op. cit]. These verifications show also that Conjecture 1.2 is true in these cases.

\section{References}

[Benard 1971] M. Benard, "On the Schur indices of characters of the exceptional Weyl groups", Ann. of Math. (2) 94 (1971), 89-107. MR 45 \#6939 Zbl 0202.02903

[Cline et al. 1996] E. Cline, B. Parshall, and L. Scott, Stratifying endomorphism algebras, Mem. Amer. Math. Soc. 591, 1996. MR 97h:16012 Zbl 0888.16006

[Curtis and Reiner 1987] C. W. Curtis and I. Reiner, Methods of representation theory, vol. 2, Wiley, New York, 1987. MR 88f:20002 Zbl 0616.20001

[Deng et al. 2008] B. Deng, J. Du, B. Parshall, and J. Wang, Finite dimensional algebras and quantum groups, Mathematical Surveys and Monographs 150, American Mathematical Society, Providence, RI, 2008. MR 2009i:17023 Zbl 1154.17003

[Du and Scott 1994] J. Du and L. Scott, "Lusztig conjectures, old and new, I", J. Reine Angew. Math. 455 (1994), 141-182. MR 95i:20062 Zbl 0840.17009

[Du et al. 1998] J. Du, B. Parshall, and L. Scott, "Stratifying endomorphism algebras associated to Hecke algebras”, J. Algebra 203:1 (1998), 169-210. MR 99e:20006 Zbl 0920.20008

[Ginzburg et al. 2003] V. Ginzburg, N. Guay, E. Opdam, and R. Rouquier, "On the category $\mathcal{O}$ for rational Cherednik algebras”, Invent. Math. 154:3 (2003), 617-651. MR 2005f:20010 Zbl 1071.20005

[Lusztig 1984] G. Lusztig, Characters of reductive groups over a finite field, Annals of Mathematics Studies 107, Princeton University Press, 1984. MR 86j:20038 Zbl 0556.20033

[Lusztig 1985] G. Lusztig, "Cells in affine Weyl groups”, pp. 255-287 in Algebraic groups and related topics (Kyoto/Nagoya, 1983), edited by R. Hotta, Adv. Stud. Pure Math. 6, North-Holland, Amsterdam, 1985. MR 87h:20074 Zbl 0569.20032

[Lusztig 1987a] G. Lusztig, "Cells in affine Weyl groups, II”, J. Algebra 109:2 (1987), 536-548. MR 88m:20103a Zbl 0625.20032 
[Lusztig 1987b] G. Lusztig, "Cells in affine Weyl groups, III", J. Fac. Sci. Univ. Tokyo Sect. IA Math. 34:2 (1987), 223-243. MR 88m:20103b Zbl 0631.20028

[Lusztig 2003] G. Lusztig, Hecke algebras with unequal parameters, CRM Monograph Series 18, American Mathematical Society, Providence, RI, 2003. MR 2004k:20011 Zbl 1051.20003

[Rouquier 2008] R. Rouquier, " $q$-Schur algebras and complex reflection groups", Mosc. Math. J. 8:1 (2008), 119-158. MR 2010b:20081 Zbl 1213.20007

Received June 15, 2015. Revised September 25, 2015.

JIE DU

SCHOOL OF MATHEMATICS AND STATISTICS

UNIVERSITY OF NEW SOUTH WALES

SYDNEY, NSW 2052

Australia

j.du@unsw.edu.au

BRIAN J. PARSHALL

DEPARTMENT OF MATHEMATICS

UNIVERSITY OF VIRGINIA

CHARLOTTESVILle, VA 22903

UNITED STATES

bjp8w@virginia.edu

LEONARD L. SCOTT

DEPARTMENT OF MATHEMATICS

UNIVERSITY OF VIRGINIA

CharlotTESVILle, VA 22903

UNITED STATES

1ls21@virginia.edu 


\title{
PACIFIC JOURNAL OF MATHEMATICS
}

\author{
msp.org/pjm
}

Founded in 1951 by E. F. Beckenbach (1906-1982) and F. Wolf (1904-1989)

\section{EDITORS}

Don Blasius (Managing Editor)

Department of Mathematics

University of California

Los Angeles, CA 90095-1555

blasius@math.ucla.edu

\author{
Paul Balmer \\ Department of Mathematics \\ University of California \\ Los Angeles, CA 90095-1555 \\ balmer@math.ucla.edu \\ Robert Finn \\ Department of Mathematics \\ Stanford University \\ Stanford, CA 94305-2125 \\ finn@math.stanford.edu \\ Sorin Popa \\ Department of Mathematics \\ University of California \\ Los Angeles, CA 90095-1555 \\ popa@math.ucla.edu
}

\author{
Vyjayanthi Chari \\ Department of Mathematics \\ University of California \\ Riverside, CA 92521-0135 \\ chari@math.ucr.edu \\ Kefeng Liu \\ Department of Mathematics \\ University of California \\ Los Angeles, CA 90095-1555 \\ liu@math.ucla.edu \\ Jie Qing \\ Department of Mathematics \\ University of California \\ Santa Cruz, CA 95064 \\ qing@ cats.ucsc.edu
}

\section{PRODUCTION}

Silvio Levy, Scientific Editor, production@msp.org

\section{SUPPORTING INSTITUTIONS}

ACADEMIA SINICA, TAIPEI

CALIFORNIA INST. OF TECHNOLOGY

INST. DE MATEMÁTICA PURA E APLICADA

KEIO UNIVERSITY

MATH. SCIENCES RESEARCH INSTITUTE

NEW MEXICO STATE UNIV.

OREGON STATE UNIV.

\author{
STANFORD UNIVERSITY \\ UNIV. OF BRITISH COLUMBIA \\ UNIV. OF CALIFORNIA, BERKELEY \\ UNIV. OF CALIFORNIA, DAVIS \\ UNIV. OF CALIFORNIA, LOS ANGELES \\ UNIV. OF CALIFORNIA, RIVERSIDE \\ UNIV. OF CALIFORNIA, SAN DIEGO \\ UNIV. OF CALIF., SANTA BARBARA
}

\author{
Daryl Cooper \\ Department of Mathematics \\ University of California \\ Santa Barbara, CA 93106-3080 \\ cooper@math.ucsb.edu \\ Jiang-Hua Lu \\ Department of Mathematics \\ The University of Hong Kong \\ Pokfulam Rd., Hong Kong \\ jhlu@maths.hku.hk \\ Paul Yang \\ Department of Mathematics \\ Princeton University \\ Princeton NJ 08544-1000 \\ yang@math.princeton.edu
}

These supporting institutions contribute to the cost of publication of this Journal, but they are not owners or publishers and have no responsibility for its contents or policies.

See inside back cover or msp.org/pjm for submission instructions.

The subscription price for 2015 is US \$420/year for the electronic version, and \$570/year for print and electronic.

Subscriptions, requests for back issues and changes of subscribers address should be sent to Pacific Journal of Mathematics, P.O. Box 4163, Berkeley, CA 94704-0163, U.S.A. The Pacific Journal of Mathematics is indexed by Mathematical Reviews, Zentralblatt MATH, PASCAL CNRS Index, Referativnyi Zhurnal, Current Mathematical Publications and Web of Knowledge (Science Citation Index).

The Pacific Journal of Mathematics (ISSN 0030-8730) at the University of California, c/o Department of Mathematics, 798 Evans Hall \#3840, Berkeley, CA 94720-3840, is published twelve times a year. Periodical rate postage paid at Berkeley, CA 94704, and additional mailing offices. POSTMASTER: send address changes to Pacific Journal of Mathematics, P.O. Box 4163, Berkeley, CA 94704-0163.

PJM peer review and production are managed by EditFLOW ${ }^{\circledR}$ from Mathematical Sciences Publishers.

\section{PUBLISHED BY}

\section{mathematical sciences publishers \\ nonprofit scientific publishing}

http://msp.org/

(C) 2015 Mathematical Sciences Publishers 


\title{
PACIFIC JOURNAL OF MATHEMATICS
}

\author{
Volume 279 No. 1-2 December 2015
}

In memoriam: Robert Steinberg

Robert Steinberg (1922-2014): In memoriam V. S. VARADARAJAN

Cellularity of certain quantum endomorphism algebras

HENNING H. ANDERSEN, GUSTAV I. LEHRER and RUIBIN ZHANG

Lower bounds for essential dimensions in characteristic 2 via orthogonal representations ANTONIO BABIC and VLADIMIR CHERNOUSOV

Cocharacter-closure and spherical buildings

Michael Bate, Sebastian Herpel, Benjamin Martin and Gerhard RöHrLe

Embedding functor for classical groups and Brauer-Manin obstruction

Eva Bayer-Fluckiger, Ting-Yu LeE and Raman Parimala

On maximal tori of algebraic groups of type $G_{2}$

Constantin Beli, PhilipPe Gille and Ting-Yu LeE

On extensions of algebraic groups with finite quotient

MICHEL BRION

Essential dimension and error-correcting codes

SHANE CERNELE and ZiNOVy REICHSTEIN

Notes on the structure constants of Hecke algebras of induced representations of finite Chevalley groups

Charles W. CuRTis

Complements on disconnected reductive groups

FRANÇOIS DIGNE and JEAN MICHEL

Extending Hecke endomorphism algebras

Jie Du, Brian J. Parshall and LeOnard L. SCOTT

Products of partial normal subgroups

ELLEN HENKE

Lusztig induction and $\ell$-blocks of finite reductive groups

RADHA KESSAR and GUNTER MALLE

Free resolutions of some Schubert singularities

Manoj Kummini, Venkatramani Lakshmibai, Pramathanath Sastry and C. S. Seshadri

Free resolutions of some Schubert singularities in the Lagrangian Grassmannian

VenKatramani LAKSHMibai and ReUVEN HODGES

Distinguished unipotent elements and multiplicity-free subgroups of simple algebraic groups

Martin W. Liebeck, Gary M. Seitz and Donna M. Testerman

Action of longest element on a Hecke algebra cell module

GEORGE LUSZTIG

Generic stabilisers for actions of reductive groups

BENJAMIN MARTIN

On the equations defining affine algebraic groups

VLADIMIR L. POPOV

Smooth representations and Hecke modules in characteristic $p$

PETER SCHNEIDER

On CRDAHA and finite general linear and unitary groups

BHAMA SRINIVASAN

Weil representations of finite general linear groups and finite special linear groups PHAM HUU TIEP

The pro- $p$ Iwahori Hecke algebra of a reductive $p$-adic group, $\mathrm{V}$ (parabolic induction) MARIE-FRANCE VIGNÉRAS

Acknowledgement 